\title{
WEIGHTED COMPOSITION OPERATORS ON BMOA
}

\author{
JUSSI LAITILA
}

\begin{abstract}
Let $\psi$ and $\varphi$ be analytic functions on the unit disk $\mathbb{D}$ such that $\varphi(\mathbb{D}) \subset \mathbb{D}$. We characterize boundedness and compactness of the weighted composition operators $f \mapsto \psi \cdot(f \circ \varphi)$ on BMOA, the space of analytic functions on the unit disk that have bounded mean oscillation on the unit circle, and its subspace VMOA. We also estimate the essential norm of a weighted composition operator on VMOA.
\end{abstract}

\section{INTRODUCTION}

Let $\mathbb{D}$ be the open unit disk in the complex plane and let $\psi: \mathbb{D} \rightarrow \mathbb{C}$ and $\varphi: \mathbb{D} \rightarrow \mathbb{D}$ be analytic functions. The weighted composition operator $W_{\psi, \varphi}$ is the linear operator defined on $\mathcal{H}(\mathbb{D})$, the linear space of all analytic functions on $\mathbb{D}$, by

$$
\left(W_{\psi, \varphi} f\right)(z)=\psi(z) f(\varphi(z)), \quad z \in \mathbb{D},
$$

for all $f \in \mathcal{H}(\mathbb{D})$. This operator can be viewed as a simultaneous generalization of both the pointwise multiplier $M_{\psi}: f \mapsto \psi \cdot f$ and the composition operator $C_{\varphi}: f \mapsto f \circ \varphi$. Weighted composition operators appear in various settings in the literature. For example, it is known that isometries of many analytic function spaces are weighted composition operators (see [FJ, §4], for instance). Recently boundedness and compactness of weighted composition operators have been studied on various classical Banach spaces of analytic functions on $\mathbb{D}$, such as Hardy, Bergman, and Bloch spaces, see e.g. $[\mathrm{CH}]$,

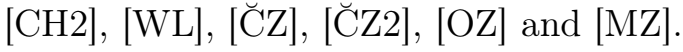

The purpose of this paper is to consider the weighted composition operators $W_{\psi, \varphi}$ on the space BMOA, which consists of the analytic functions on $\mathbb{D}$ that have bounded mean oscillation on the unit circle $\mathbb{T}$. Our main goal is to characterize boundedness and compactness of the operators $W_{\psi, \varphi}$ on $\mathrm{BMOA}$ in terms of function theoretic properties of the symbols $\psi$ and $\varphi$. We also characterize boundedness and compactness of $W_{\psi, \varphi}$ on VMOA, the closed subspace of BMOA consisting of the analytic functions of vanishing mean oscillation. Moreover, we estimate the essential norm (that is, the distance to all compact operators) of a weighted composition operator $W_{\psi, \varphi}$ on VMOA. Our estimates appear to be new also in the special cases of the operators $M_{\psi}$ and $C_{\varphi}$.

Date: 26th June 2006.

2000 Mathematics Subject Classification. Primary: 47B38, Secondary: 30H05, 30D50.

Key words and phrases. Weighted composition operator, bounded mean oscillation.

The author was supported in part by the Finnish Academy of Science and Letters (Vilho, Yrjö and Kalle Väisälä Foundation) and the Academy of Finland, projects \#53893 and \#210970. 
Boundedness and compactness of the operators $M_{\psi}$ and $C_{\varphi}$ are quite well understood in the context of BMOA. Boundedness of $M_{\psi}$ on BMOA was first characterized by Stegenga [St]. In fact, $M_{\psi}$ is bounded if and only if $\psi$ is bounded and has logarithmic mean oscillation. It is not difficult to see that $M_{\psi}$ can be compact on BMOA only if $\psi \equiv 0$. The classical Littlewood subordination principle implies that every composition operator $C_{\varphi}$ is bounded on the Hardy space $H^{2}$. It follows from this result that every composition operator is bounded also on BMOA (see e.g. [Ste], [AFP]). Compactness of composition operators on BMOA and VMOA has been studied (and characterized) by several authors, see e.g. [T], [BCM], [Sm] and [MT]. We refer to the monographs $[\mathrm{S} 2]$ and $[\mathrm{CoM}]$ for the basic results about composition operators $C_{\varphi}$ on classical spaces of analytic functions on $\mathbb{D}$.

This paper is organized as follows. In Sections 3 and 4 we characterize boundedness and compactness of the operators $W_{\psi, \varphi}$ on BMOA. These results are based on a weighted version of the Littlewood subordination principle which will also be established in Section 3. In Section 5 we study boundedness, compactness and the essential norm of $W_{\psi, \varphi}$ on VMOA. In Section 6 we exhibit some concrete examples of bounded and compact weighted composition operators on BMOA and VMOA. In the final Section 7 we compare the boundedness and compactness results on BMOA and the Bloch space $\mathcal{B}$ (due to Ohno and Zhao [OZ]). In particular, we show that the bounded (respectively compact) weighted composition operators on BMOA are bounded (respectively compact) on $\mathcal{B}$.

\section{Preliminaries}

Let $\mathbb{D}=\{z \in \mathbb{C}:|z|<1\}$ be the open unit disk in $\mathbb{C}$ and let $\mathbb{T}=\partial \mathbb{D}$ be the unit circle. Recall that a function $f \in \mathcal{H}(\mathbb{D})$ belongs to the space BMOA if and only if it is a Poisson extension of some function on $\mathbb{T}$ that has bounded mean oscillation. There are many ways to define a complete norm on BMOA. We will view BMOA as a Möbius invariant version of the Hardy space $H^{2}$ as follows. For $1 \leq p<\infty$, let $H^{p}$ denote the usual Hardy space of functions $g \in \mathcal{H}(\mathbb{D})$ with the norm

$$
\|g\|_{H^{p}}=\left(\int_{\mathbb{T}}|g(\zeta)|^{p} d m(\zeta)\right)^{1 / p},
$$

where $g(\zeta)=\lim _{r \rightarrow 1} g(r \zeta)$ is the almost everywhere on $\mathbb{T}$ existing radial limit and $m$ is the Lebesgue measure on $\mathbb{T}$ normalized so that $m(\mathbb{T})=1$. Then $f \in$ BMOA if and only if $f \in \mathcal{H}(\mathbb{D})$ and

$$
\|f\|_{*}=\sup _{a \in \mathbb{D}}\left\|f \circ \sigma_{a}-f(a)\right\|_{H^{2}}<\infty
$$

where the automorphisms $\sigma_{a}: \mathbb{D} \rightarrow \mathbb{D}$ are given by $\sigma_{a}(z)=(a-z) /(1-\bar{a} z)$ for $a, z \in \mathbb{D}$ (see $[\mathrm{B}])$. The quantity $\|f\|_{*}$ is a seminorm and $\|f\|_{\mathrm{BMOA}}=$ $|f(0)|+\|f\|_{*}$ defines a complete norm on BMOA.

We recall next some basic properties of BMOA functions which will be needed later. Note first that $\|f\|_{H^{2}} \leq\|f\|_{\mathrm{BMOA}}$ for all $f \in \mathrm{BMOA}$ and $\|f\|_{\text {BMOA }} \leq 3\|f\|_{\infty}$ for $f \in H^{\infty}$, where $\|f\|_{\infty}=\sup _{z \in \mathbb{D}}|f(z)|$. By the 
Hölder inequality, we have that

$$
\|f\|_{*} \leq \sup _{a \in \mathbb{D}}\left\|f \circ \sigma_{a}-f(a)\right\|_{H^{p}},
$$

for $2 \leq p<\infty$. We will need the remarkable fact that for each $2 \leq p<\infty$ this inequality can be reversed. In fact, it follows from the John-Nirenberg theorem (see [B, Corollary 3], [G, §VI.2]) that given $2 \leq p<\infty$, there is a constant $K_{p}$ such that

$$
\sup _{a \in \mathbb{D}}\left\|f \circ \sigma_{a}-f(a)\right\|_{H^{p}} \leq K_{p}\|f\|_{*},
$$

for every $f \in$ BMOA. (In fact, for each $0<p<\infty$, the quantity on the left-hand side of (2.1) defines an equivalent seminorm on BMOA, see [B].)

We establish next some known pointwise estimates for BMOA functions. One verifies from the Cauchy integral formula for the derivative that $\left|f^{\prime}(0)\right| \leq$ $\|f\|_{H^{2}}$ for all $f \in H^{2}$. Therefore

$$
\left(1-|a|^{2}\right)\left|f^{\prime}(a)\right|=\left|\left(f \circ \sigma_{a}-f(a)\right)^{\prime}(0)\right| \leq\|f\|_{*},
$$

for $f \in$ BMOA. Hence BMOA $\subset \mathcal{B}$, where $\mathcal{B}$ is the Bloch space which consists of all $f \in \mathcal{H}(\mathbb{D})$ such that $\|f\|_{\mathcal{B}}=|f(0)|+\sup _{z \in \mathbb{D}}\left(1-|z|^{2}\right)\left|f^{\prime}(z)\right|<\infty$. From a well-known pointwise estimate for the Bloch functions $[\mathrm{Z}$, Theorem 5.1.6] one gets that

$$
|f(z)-f(0)| \leq \frac{1}{2}\|f\|_{*} \log \frac{1+|z|}{1-|z|},
$$

for $f \in$ BMOA, $z \in \mathbb{D}$. Consequently,

$$
|f(z)| \leq \frac{1}{\log 2}\left(\log \frac{2}{1-|z|^{2}}\right)\|f\|_{\mathrm{BMOA}}
$$

for $f \in \mathrm{BMOA}, z \in \mathbb{D}$. We refer to $[\mathrm{G}]$ and $[\mathrm{Z}]$ for further properties of BMOA functions.

\section{Boundedness of $W_{\psi, \varphi}$ ON BMOA}

In this section we characterize boundedness of the weighted composition operators $W_{\psi, \varphi}: f \mapsto \psi \cdot(f \circ \varphi)$ on BMOA. It is convenient to introduce the following abbreviations which will be used throughout the paper. Let

$$
\alpha(\psi, \varphi, a)=|\psi(a)| \cdot\left\|\sigma_{\varphi(a)} \circ \varphi \circ \sigma_{a}\right\|_{H^{2}}
$$

and

$$
\beta(\psi, \varphi, a)=\left(\log \frac{2}{1-|\varphi(a)|^{2}}\right)\left\|\psi \circ \sigma_{a}-\psi(a)\right\|_{H^{2}},
$$

for $a \in \mathbb{D}$ and analytic functions $\varphi: \mathbb{D} \rightarrow \mathbb{D}, \psi: \mathbb{D} \rightarrow \mathbb{C}$. Moreover, given any Banach space $E$, we denote by $B_{E}=\left\{x \in E:\|x\|_{E} \leq 1\right\}$ the closed unit ball in $E$. We write $A \sim B$ whenever the two quantities $A$ and $B$ are comparable, i.e., there is a positive constant $C$ such that $C^{-1} B \leq A \leq C B$.

Theorem 3.1. Let $\varphi: \mathbb{D} \rightarrow \mathbb{D}$ and $\psi: \mathbb{D} \rightarrow \mathbb{C}$ be analytic. Then $W_{\psi, \varphi}$ is bounded on BMOA if and only if

$$
\sup _{a \in \mathbb{D}} \alpha(\psi, \varphi, a)<\infty \quad \text { and } \quad \sup _{a \in \mathbb{D}} \beta(\psi, \varphi, a)<\infty .
$$


Moreover,

$$
\sup _{f \in B_{\mathrm{BMOA}}}\left\|W_{\psi, \varphi} f\right\|_{*} \sim \sup _{a \in \mathbb{D}} \alpha(\psi, \varphi, a)+\sup _{a \in \mathbb{D}} \beta(\psi, \varphi, a) .
$$

We need some auxiliary results before the proof of Theorem 3.1. Recall first that if $\lambda: \mathbb{D} \rightarrow \mathbb{D}$ is an analytic map with $\lambda(0)=0$ then the Littlewood subordination principle (see [CoM, p. 30]) states that

$$
\|g \circ \lambda\|_{H^{2}} \leq\|g\|_{H^{2}},
$$

for all $g \in H^{2}$. The well-known fact that every composition operator $C_{\varphi}: f \mapsto f \circ \varphi$ is bounded BMOA (see e.g. [Ste, Theorem 3], [AFP, Theorem 12]) can be deduced from (3.2). Indeed, if we set $g_{a}=f \circ \sigma_{\varphi(a)}-f(\varphi(a))$ and $\lambda_{a}=\sigma_{\varphi(a)} \circ \varphi \circ \sigma_{a}$, where $f \in$ BMOA and $\varphi: \mathbb{D} \rightarrow \mathbb{D}$ are analytic, $a \in \mathbb{D}$, then

$$
\|f \circ \varphi\|_{*}=\sup _{a \in \mathbb{D}}\left\|g_{a} \circ \lambda_{a}\right\|_{H^{2}} \leq \sup _{a \in \mathbb{D}}\left\|g_{a}\right\|_{H^{2}} \leq\|f\|_{*},
$$

by (3.2) and the fact that $\left(\sigma_{\varphi(a)} \circ \sigma_{\varphi(a)}\right)(z)=z$. Hence $C_{\varphi}$ is bounded on BMOA. The following result, which provides a weighted counterpart of (3.3) will be crucial in the proof of Theorem 3.1.

Proposition 3.2. There is a constant $C>0$ such that

$$
\|g \circ \lambda\|_{H^{2}} \leq C\|\lambda\|_{H^{2}}\|g\|_{H^{2}},
$$

for all analytic functions $g \in H^{2}$ and $\lambda: \mathbb{D} \rightarrow \mathbb{D}$ such that $g(0)=\lambda(0)=0$.

In particular,

$$
|\psi(a)| \cdot\left\|f \circ \varphi \circ \sigma_{a}-f(\varphi(a))\right\|_{H^{2}} \leq C \alpha(\psi, \varphi, a)\|f\|_{*},
$$

for all $a \in \mathbb{D}$ and analytic functions $f \in \mathrm{BMOA}, \psi \in \mathcal{H}(\mathbb{D})$ and $\varphi: \mathbb{D} \rightarrow \mathbb{D}$.

The proof of Proposition 3.2 will be based on properties of the Nevanlinna counting function $N(\varphi, \cdot)$. Recall that for an analytic map $\varphi: \mathbb{D} \rightarrow \mathbb{D}$, the Nevanlinna counting function is given by $N(\varphi, w)=\sum_{z \in \varphi^{-1}(w)} \log (1 /|z|)$ for $w \in \mathbb{D} \backslash\{\varphi(0)\}$, where each point in the preimage $\varphi^{-1}(w)$ is counted according to its multiplicity. If $\varphi(0)=0$, then the Littlewood inequality states that $N(\varphi, w) \leq \log (1 /|w|)$ for $w \in \mathbb{D} \backslash\{0\}$ (see [CoM, p. 33]). The following result due to W. Smith provides a weighted version of this inequality.

Lemma 3.3 ([Sm, Lemma 2.1]). Let $\lambda: \mathbb{D} \rightarrow \mathbb{D}$ be an analytic map with $\lambda(0)=0$ and let $\gamma(\lambda)=\sup \left\{|w|^{2} N(\lambda, w): w \in \mathbb{D} \backslash\{0\}\right\}$. Then

$$
N(\lambda, z) \leq \frac{4}{\log 2} \gamma(\lambda) \log \frac{1}{|z|},
$$

for all $z \in \mathbb{D}$ such that $\frac{1}{2} \leq|z|<1$.

The relevance of the Nevanlinna counting function is seen from the change of variable formula

$$
\int_{\mathbb{D}}\left|(f \circ \varphi)^{\prime}(w)\right|^{2} \log \frac{1}{|w|} \frac{d A(w)}{\pi}=\int_{\mathbb{D}}\left|f^{\prime}(z)\right|^{2} N(\varphi, z) \frac{d A(z)}{\pi},
$$


which holds for all $f \in H^{2}$ and analytic mappings $\varphi: \mathbb{D} \rightarrow \mathbb{D}$ (see $[\mathrm{CoM}$, p. 35]). Here $A$ is the Lebesgue area measure on $\mathbb{D}$. Combined with the Littlewood-Paley identity (see [CoM, p. 34] or [G, p. 236])

$$
\|f-f(0)\|_{H^{2}}^{2}=2 \int_{\mathbb{D}}\left|f^{\prime}(w)\right|^{2} \log \frac{1}{|w|} \frac{d A(w)}{\pi},
$$

formula (3.6) yields the fundamental identity

$$
\|f \circ \varphi-f(\varphi(0))\|_{H^{2}}^{2}=2 \int_{\mathbb{D}}\left|f^{\prime}(z)\right|^{2} N(\varphi, z) \frac{d A(z)}{\pi},
$$

for all $f \in H^{2}$ and analytic mappings $\varphi: \mathbb{D} \rightarrow \mathbb{D}$.

We next prove Proposition 3.2.

Proof of Proposition 3.2. We first establish the estimate

$$
N(\lambda, z) \leq \frac{8}{\log 2}\|\lambda\|_{H^{2}}^{2} \log \frac{1}{|z|}
$$

for $z \in \mathbb{D} \backslash \frac{1}{2} \mathbb{D}$ and analytic functions $\lambda: \mathbb{D} \rightarrow \mathbb{D}$ such that $\lambda(0)=0$. A simple calculation shows that

$$
\left\|\sigma_{w} \circ \lambda-\sigma_{w}(\lambda(0))\right\|_{H^{2}}^{2}=\int_{\mathbb{T}} \frac{\left(1-|w|^{2}\right)^{2}|\lambda(\zeta)|^{2}}{|1-\bar{w} \lambda(\zeta)|^{2}} d m(\zeta) \leq 4\|\lambda\|_{H^{2}}^{2},
$$

for $w \in \mathbb{D}$. Since $\left(\sigma_{w} \circ \lambda\right)(0)=w \notin t|w| \mathbb{D}$ for $w \in \mathbb{D} \backslash\{0\}$ and $t \in(0,1)$, we get from the sub-mean value property of the Nevanlinna counting function (see [CoM, p. 137]) and (3.8) that

$$
t^{2}|w|^{2} N\left(\sigma_{w} \circ \lambda, 0\right) \leq \int_{t|w| \mathbb{D}} N\left(\sigma_{w} \circ \lambda, z\right) \frac{d A(z)}{\pi} \leq 2\|\lambda\|_{H^{2}}^{2},
$$

for all $t \in(0,1)$. It is easy to verify that $N(\lambda, w)=N\left(\sigma_{w} \circ \lambda, 0\right)$. Hence, by letting $t \rightarrow 1$ and taking the supremum over $w \in \mathbb{D} \backslash\{0\}$ in (3.10), we get that

$$
\sup _{w \in \mathbb{D} \backslash\{0\}}|w|^{2} N(\lambda, w) \leq 2\|\lambda\|_{H^{2}}^{2} .
$$

The estimate (3.9) follows now from Lemma 3.3.

Assume next that $g \in H^{2}$ satisfies $g(0)=0$. Then $g(\lambda(0))=0$ so that

$$
\|g \circ \lambda\|_{H^{2}}^{2}=2 \int_{\mathbb{D}}\left|g^{\prime}(z)\right|^{2} N(\lambda, z) \frac{d A(z)}{\pi},
$$

by (3.8). By applying (3.9) and (3.7) we get that

$$
\begin{aligned}
\int_{\mathbb{D} \backslash \frac{1}{2} \mathbb{D}}\left|g^{\prime}(z)\right|^{2} N(\lambda, z) \frac{d A(z)}{\pi} & \leq \frac{8}{\log 2}\|\lambda\|_{H^{2}}^{2} \int_{\mathbb{D} \backslash \frac{1}{2} \mathbb{D}}\left|g^{\prime}(z)\right|^{2} \log \frac{1}{|z|} \frac{d A(z)}{\pi} \\
& \leq(4 / \log 2)\|g\|_{H^{2}}^{2}\|\lambda\|_{H^{2}}^{2} .
\end{aligned}
$$

On the other hand, since $\left|g^{\prime}(z)\right| \leq(1-|z|)^{-2}\|g\|_{H^{2}}$ for $z \in \mathbb{D}$, by the Cauchy integral formula and the Hölder inequality, we get that

$$
\int_{\frac{1}{2} \mathbb{D}}\left|g^{\prime}(z)\right|^{2} N(\lambda, z) \frac{d A(z)}{\pi} \leq 16\|g\|_{H^{2}}^{2} \int_{\frac{1}{2} \mathbb{D}} N(\lambda, z) \frac{d A(z)}{\pi} \leq 8\|g\|_{H^{2}}^{2}\|\lambda\|_{H^{2}}^{2} .
$$

By combining these estimates with (3.12), one obtains that $\|g \circ \lambda\|_{H^{2}}^{2} \leq$ $(8 / \log 2+16)\|g\|_{H^{2}}^{2}\|\lambda\|_{H^{2}}^{2}$. This proves (3.4). 
Let finally $f \in$ BMOA, $\psi \in \mathcal{H}(\mathbb{D})$ and $\varphi: \mathbb{D} \rightarrow \mathbb{D}$ be arbitrary analytic functions and put $g_{a}=f \circ \sigma_{\varphi(a)}-f(\varphi(a))$ and $\lambda_{a}=\sigma_{\varphi(a)} \circ \varphi \circ \sigma_{a}$ for $a \in \mathbb{D}$. Clearly $\lambda_{a}(\mathbb{D}) \subset \mathbb{D}$ and $g_{a}(0)=\lambda_{a}(0)=0$. Since $\left\|g_{a}\right\|_{H^{2}} \leq\|f\|_{*}$, we get from (3.4) that $\left\|f \circ \varphi \circ \sigma_{a}-f(\varphi(a))\right\|_{H^{2}}=\left\|g_{a} \circ \lambda_{a}\right\|_{H^{2}} \leq C\left\|\lambda_{a}\right\|_{H^{2}}\|f\|_{*}$. We obtain (3.5) by multiplying both sides of this inequality by $|\psi(a)|$.

We are now ready to prove Theorem 3.1.

Proof of Theorem 3.1. Suppose first that $W_{\psi, \varphi}$ is bounded on BMOA, so that $T:=\sup \left\{\left\|W_{\psi, \varphi} f\right\|_{*}: f \in B_{\mathrm{BMOA}}\right\}<\infty$. Since $\psi=W_{\psi, \varphi}(1)$, we have that $\psi \in$ BMOA with $\|\psi\|_{*} \leq T$. Put

$$
f_{a}(z)=\sigma_{\varphi(a)}(z)-\varphi(a)=\frac{\left(|\varphi(a)|^{2}-1\right) z}{1-\overline{\varphi(a)} z},
$$

for $a, z \in \mathbb{D}$. Then $f_{a}(0)=0, f_{a}(\varphi(a))=-\varphi(a)$ and $\left\|f_{a}\right\|_{\infty} \leq 2$. Moreover, $\left\|f_{a}\right\|_{\mathrm{BMOA}}=\left\|\sigma_{\varphi(a)}\right\|_{*} \leq 1$ and $\sigma_{\varphi(a)} \circ \varphi \circ \sigma_{a}=f_{a} \circ \varphi \circ \sigma_{a}-f_{a}(\varphi(a))$. By adding and subtracting the term $\left(\psi \circ \sigma_{a}\right) \cdot f_{a} \circ \varphi \circ \sigma_{a}$ and applying the triangle inequality, we get that

$$
\begin{aligned}
\alpha(\psi, \varphi, a)= & \left\|\psi(a)\left(f_{a} \circ \varphi \circ \sigma_{a}-f_{a}(\varphi(a))\right)\right\|_{H^{2}} \\
\leq & \left\|\left(\psi(a)-\psi \circ \sigma_{a}\right) \cdot f_{a} \circ \varphi \circ \sigma_{a}\right\|_{H^{2}} \\
& +\left\|\left(\psi \circ \sigma_{a}\right) \cdot f_{a} \circ \varphi \circ \sigma_{a}-\psi(a) f_{a}(\varphi(a))\right\|_{H^{2}} \\
\leq & \left\|\psi \circ \sigma_{a}-\psi(a)\right\|_{H^{2}}\left\|f_{a}\right\|_{\infty}+\left\|W_{\psi, \varphi} f_{a}\right\|_{*} \leq 3 T .
\end{aligned}
$$

Consider next the analytic functions

$$
g_{a}(z)=\log \left(\frac{2}{1-\overline{\varphi(a)} z}\right),
$$

for $a, z \in \mathbb{D}$. Recall that $g_{a} \in$ BMOA with $M:=\sup _{a \in \mathbb{D}}\left\|g_{a}\right\|_{*}<\infty$. By adding and subtracting the term $\left(\psi \circ \sigma_{a}\right) \cdot\left(g_{a} \circ \varphi \circ \sigma_{a}\right)-\psi(a) \cdot\left(g_{a} \circ \varphi \circ \sigma_{a}\right)+$ $\psi(a) g_{a}(\varphi(a))$ and applying the triangle inequality again, we get that

$$
\begin{aligned}
\beta(\psi, \varphi, a)= & \left\|\left(\psi \circ \sigma_{a}-\psi(a)\right) g_{a}(\varphi(a))\right\|_{H^{2}} \\
\leq & \left\|\left(\psi \circ \sigma_{a}-\psi(a)\right) \cdot\left(g_{a}(\varphi(a))-g_{a} \circ \varphi \circ \sigma_{a}\right)\right\|_{H^{2}} \\
& +\left\|\left(\psi \circ \sigma_{a}\right) \cdot g_{a} \circ \varphi \circ \sigma_{a}-\psi(a) g_{a}(\varphi(a))\right\|_{H^{2}} \\
& +\left\|\psi(a)\left(g_{a}(\varphi(a))-g_{a} \circ \varphi \circ \sigma_{a}\right)\right\|_{H^{2}} .
\end{aligned}
$$

The Hölder inequality, reverse Hölder inequality (2.1), and (3.3) give that

$$
\begin{aligned}
\|\left(\psi \circ \sigma_{a}-\psi(a)\right) & \cdot\left(g_{a}(\varphi(a))-g_{a} \circ \varphi \circ \sigma_{a}\right) \|_{H^{2}} \\
& \leq\left\|\psi \circ \sigma_{a}-\psi(a)\right\|_{H^{4}}\left\|g_{a} \circ \varphi \circ \sigma_{a}-g_{a}(\varphi(a))\right\|_{H^{4}} \\
& \leq K_{4}^{2}\|\psi\|_{*}\left\|g_{a} \circ \varphi\right\|_{*} \leq K_{4}^{2} T M .
\end{aligned}
$$

Hence we get from (3.5) and (3.14) that

$$
\begin{aligned}
\beta(\psi, \varphi, a) & \leq K_{4}^{2} T M+\left\|W_{\psi, \varphi} g_{a}\right\|_{*}+C \alpha(\psi, \varphi, a)\left\|g_{a}\right\|_{*} \\
& \leq M\left(K_{4}^{2}+1+3 C\right) T .
\end{aligned}
$$


By combining (3.14) and (3.17), and taking the supremum over $a \in \mathbb{D}$, we get the desired estimate

$$
C_{1}\left(\sup _{a \in \mathbb{D}} \alpha(\psi, \varphi, a)+\sup _{a \in \mathbb{D}} \beta(\psi, \varphi, a)\right) \leq \sup _{f \in B_{\mathrm{BMOA}}}\left\|W_{\psi, \varphi} f\right\|_{*},
$$

for a suitable constant $C_{1}>0$.

Suppose next that (3.1) holds. Since $\log 2 \leq \log \left(2 /\left(1-|\varphi(a)|^{2}\right)\right)$, we have that

$$
\left\|\psi \circ \sigma_{a}-\psi(a)\right\|_{H^{2}} \leq(\log 2)^{-1} \beta(\psi, \varphi, a),
$$

so that $\psi \in \mathrm{BMOA}$. Let $f \in B_{\mathrm{BMOA}}$ be arbitrary. Then $\left\|W_{\psi, \varphi} f\right\|_{\mathrm{BMOA}}=$ $|\psi(0) f(\varphi(0))|+\sup _{a \in \mathbb{D}}\left\|\left(\psi \circ \sigma_{a}\right) \cdot f \circ \varphi \circ \sigma_{a}-\psi(a) f(\varphi(a))\right\|_{H^{2}}$, where the term $|\psi(0) f(\varphi(0))|$ is bounded, by (2.2). By applying the triangle inequality we get that

$$
\begin{aligned}
&\left\|\left(\psi \circ \sigma_{a}\right) \cdot f \circ \varphi \circ \sigma_{a}-\psi(a) f(\varphi(a))\right\|_{H^{2}} \\
& \leq\left\|\left(\psi \circ \sigma_{a}-\psi(a)\right) \cdot\left(f \circ \varphi \circ \sigma_{a}-f(\varphi(a))\right)\right\|_{H^{2}} \\
&+\left\|\psi(a) \cdot\left(f \circ \varphi \circ \sigma_{a}-f(\varphi(a))\right)\right\|_{H^{2}} \\
&+\left\|\left(\psi \circ \sigma_{a}-\psi(a)\right) \cdot f(\varphi(a))\right\|_{H^{2}} .
\end{aligned}
$$

Hence we get from (2.1), (3.5) and (2.2) that

$$
\left\|W_{\psi, \varphi} f\right\|_{*} \leq K_{4}^{2}\|\psi\|_{*}\|f \circ \varphi\|_{*}+\sup _{a \in \mathbb{D}}\left(C \alpha(\psi, \varphi, a)+(\log 2)^{-1} \beta(\psi, \varphi, a)\right),
$$

where

$$
\|\psi\|_{*}\|f \circ \varphi\|_{*} \leq(\log 2)^{-1} \sup _{a \in \mathbb{D}} \beta(\psi, \varphi, a),
$$

by (3.18) and (3.3). Hence $W_{\psi, \varphi}$ is bounded on BMOA and

$$
\sup _{f \in B_{\mathrm{BMOA}}}\left\|W_{\psi, \varphi} f\right\|_{*} \leq C_{2}\left(\sup _{a \in \mathbb{D}} \alpha(\psi, \varphi, a)+\sup _{a \in \mathbb{D}} \beta(\psi, \varphi, a)\right),
$$

for some constant $C_{2}>0$. This completes the proof of Theorem 3.1.

Theorem 3.1 contains as a special case the well-known LMOA condition which characterizes boundedness of pointwise multipliers $M_{\psi}$ on BMOA (see [St, Theorem 1.2] or [OF, Theorem A]). There are several equivalent formulations of LMOA. We say that a function $\psi \in \mathcal{H}(\mathbb{D})$ has logarithmic mean oscillation (denoted by $\psi \in \mathrm{LMOA}$ ) if

$$
\|\psi\|_{*, \log }:=\sup _{a \in \mathbb{D}}\left(\log \frac{2}{1-|a|^{2}}\right)\left\|\psi \circ \sigma_{a}-\psi(a)\right\|_{H^{2}}<\infty .
$$

By letting $\varphi(z)=z$ for all $z \in \mathbb{D}$, we have $M_{\psi}=W_{\psi, \varphi}$. In this case $\sup _{a \in \mathbb{D}} \beta(\psi, \varphi, a)=\|\psi\|_{*, \log }$ and $\sup _{a \in \mathbb{D}} \alpha(\psi, \varphi, a)=\|\psi\|_{\infty}$, since $\left(\sigma_{\varphi(a)} \circ \varphi \circ\right.$ $\left.\sigma_{a}\right)(z)=z$ for all $a, z \in \mathbb{D}$. Since $\sup _{f \in B_{\mathrm{BMOA}}}\left|\left(M_{\psi} f\right)(0)\right|=|\psi(0)| \leq\|\psi\|_{\infty}$, Theorem 3.1 yields the following result.

Corollary 3.4. The pointwise multiplier $M_{\psi}$ is bounded on BMOA if and only if $\psi \in H^{\infty} \cap$ LMOA. Moreover, $\left\|M_{\psi}\right\| \sim\|\psi\|_{\infty}+\|\psi\|_{*, \log }$. 


\section{Compactness of $W_{\psi, \varphi}$ ON BMOA}

We next characterize compactness of the weighted composition operators $W_{\psi, \varphi}$ on BMOA. Recall that a linear operator $T$ on a Banach space $E$ is compact if $T\left(B_{E}\right)$ is a relatively compact subset of $E$, where $B_{E}$ is the closed unit ball of $E$.

Theorem 4.1. Let $\varphi: \mathbb{D} \rightarrow \mathbb{D}$ and $\psi: \mathbb{D} \rightarrow \mathbb{C}$ be analytic functions such that $W_{\psi, \varphi}$ is bounded on BMOA. Then $W_{\psi, \varphi}$ is compact on BMOA if and only if

$$
\begin{aligned}
& \lim _{r \rightarrow 1} \sup _{\{a \in \mathbb{D}:|\varphi(a)|>r\}} \alpha(\psi, \varphi, a)=0, \\
& \lim _{r \rightarrow 1} \sup _{\{a \in \mathbb{D}:|\varphi(a)|>r\}} \beta(\psi, \varphi, a)=0,
\end{aligned}
$$

and, for all $R \in(0,1)$,

$$
\lim _{t \rightarrow 1} \sup _{\{a \in \mathbb{D}:|\varphi(a)| \leq R\}} \int_{E(\varphi, a, t)}\left|\left(\psi \circ \sigma_{a}\right)(\zeta)\right|^{2} d m(\zeta)=0,
$$

where $E(\varphi, a, t)=\left\{\zeta \in \mathbb{T}:\left|\left(\sigma_{\varphi(a)} \circ \varphi \circ \sigma_{a}\right)(\zeta)\right|>t\right\}$.

The following technical lemma will be needed in the proof of Theorem 4.1.

Lemma 4.2. Let $\psi \in \mathrm{BMOA}$ and $\varphi: \mathbb{D} \rightarrow \mathbb{D}$ be analytic. Then there is a constant $c>0$ such that

$$
\left\|\left(\psi \circ \sigma_{a}-\psi(a)\right) \cdot\left(f \circ \varphi \circ \sigma_{a}-f(\varphi(a))\right)\right\|_{H^{2}}^{2} \leq c\|\psi\|_{*}\left\|\psi \circ \sigma_{a}-\psi(a)\right\|_{H^{2}},
$$

for all $f \in B_{\mathrm{BMOA}}$ and $a \in \mathbb{D}$.

Proof. By abbreviating $\Psi=\psi \circ \sigma_{a}-\psi(a)$ and $F=f \circ \varphi \circ \sigma_{a}-f(\varphi(a))$, and applying both the Hölder and reverse Hölder inequalities, we get that

$$
\begin{aligned}
\|\Psi F\|_{H^{2}}^{2} & =\left\|\Psi\left(\Psi F^{2}\right)\right\|_{H^{1}} \leq\|\Psi\|_{H^{2}}\left\|\Psi F^{2}\right\|_{H^{2}} \leq\|\Psi\|_{H^{2}}\|\Psi\|_{H^{4}}\|F\|_{H^{8}}^{2} \\
& \leq\left\|\psi \circ \sigma_{a}-\psi(a)\right\|_{H^{2}} K_{4}\|\psi\|_{*}\left(K_{8}\|f \circ \varphi\|_{*}\right)^{2},
\end{aligned}
$$

where the constants $K_{4}$ and $K_{8}$ are from (2.1). Since $\|f \circ \varphi\|_{*} \leq\|f\|_{*} \leq 1$ by (3.3), we can take $c=\sqrt{K_{4}} K_{8}$.

Proof of Theorem 4.1. We divide the proof into two steps. The proof is partly based on an argument due to W. Smith (see the proof of [Sm, Theorem 1.1]). We will also use some ideas from [OZ] and [MZ].

Step 1. If $W_{\psi, \varphi}$ is compact on BMOA, then (4.1), (4.2) and (4.3) hold.

Proof. Assume that $W_{\psi, \varphi}$ is compact (and bounded) on BMOA. If (4.1) does not hold, then there are $\delta>0$ and a sequence of points $a_{n} \in \mathbb{D}$ such that $\left|\varphi\left(a_{n}\right)\right| \rightarrow 1$ as $n \rightarrow \infty$ and

$$
\alpha\left(\psi, \varphi, a_{n}\right) \geq \delta>0 .
$$

for all $n \in \mathbb{N}$. Define the functions $f_{n} \in B_{\mathrm{BMOA}}$ by setting $f_{n}(z)=$ $\sigma_{\varphi\left(a_{n}\right)}(z)-\varphi\left(a_{n}\right)$ for $z \in \mathbb{D}$ and $n \in \mathbb{N}$. Then the estimate (3.14) gives that

$$
\alpha\left(\psi, \varphi, a_{n}\right) \leq 2\left\|\psi \circ \sigma_{a_{n}}-\psi\left(a_{n}\right)\right\|_{H^{2}}+\left\|W_{\psi, \varphi} f_{n}\right\|_{\mathrm{BMOA}} .
$$


Since $\sup _{a \in \mathbb{D}} \beta(\psi, \varphi, a)<\infty$ by Theorem 3.1, we have that

$$
\left\|\psi \circ \sigma_{a_{n}}-\psi\left(a_{n}\right)\right\|_{H^{2}} \leq\left(\log \frac{2}{1-\left|\varphi\left(a_{n}\right)\right|^{2}}\right)^{-1} \sup _{a \in \mathbb{D}} \beta(\psi, \varphi, a) \rightarrow 0,
$$

as $n \rightarrow \infty$. Moreover, since $f_{n} \rightarrow 0$ uniformly on compact subsets of $\mathbb{D}$ and $W_{\psi, \varphi}$ is compact, we have that $\lim _{n \rightarrow \infty}\left\|W_{\psi, \varphi} f_{n}\right\|_{\mathrm{BMOA}}=0$. Combining these facts with (4.5) gives that $\lim _{n \rightarrow \infty} \alpha\left(\psi, \varphi, a_{n}\right)=0$. This contradicts (4.4), so that (4.1) is necessary for the compactness of $W_{\psi, \varphi}$ on BMOA.

Suppose next that (4.2) does not hold. Then there exist $\delta>0$ and a sequence of points $a_{n} \in \mathbb{D}$ such that $\left|\varphi\left(a_{n}\right)\right| \rightarrow 1$ as $n \rightarrow \infty$ and

$$
\beta\left(\psi, \varphi, a_{n}\right) \geq \delta>0 \text {. }
$$

for all $n \in \mathbb{N}$. Put $h_{n}(z)=\log \left(2 /\left(1-\overline{\varphi\left(a_{n}\right)} z\right)\right)$ and

$$
g_{n}(z)=\frac{h_{n}(z)^{2}}{h_{n}\left(\varphi\left(a_{n}\right)\right)}=\left(\log \frac{2}{1-\left|\varphi\left(a_{n}\right)\right|^{2}}\right)^{-1}\left(\log \frac{2}{1-\overline{\varphi\left(a_{n}\right)} z}\right)^{2},
$$

for $z \in \mathbb{D}$ and $n \in \mathbb{N}$. Then $M:=\sup _{n \in \mathbb{N}}\left\|h_{n}\right\|_{*}<\infty$. By (3.8) we get that

$$
\begin{aligned}
\| g_{n} \circ \sigma_{a} & -g_{n}(a) \|_{H^{2}}=\left(2 \int_{\mathbb{D}}\left|g_{n}^{\prime}(z)\right|^{2} N\left(\sigma_{a}, z\right) \frac{d A(z)}{\pi}\right)^{1 / 2} \\
& =\left(\frac{4}{h_{n}\left(\varphi\left(a_{n}\right)\right)^{2}} \int_{\mathbb{D}}\left|h_{n}(z)\right|^{2}\left|h_{n}^{\prime}(z)\right|^{2} N\left(\sigma_{a}, z\right) \frac{d A(z)}{\pi}\right)^{1 / 2} \\
& \leq \frac{2 M}{h_{n}\left(\varphi\left(a_{n}\right)\right)} \sup _{z \in \mathbb{D}}\left|h_{n}(z)\right| .
\end{aligned}
$$

Since $\left|h_{n}(z)\right| \leq \log \left(2 /\left(1-\left|\varphi\left(a_{n}\right)\right|\right)\right)=h_{n}\left(\varphi\left(a_{n}\right)\right)+\log \left(1+\left|\varphi\left(a_{n}\right)\right|\right)$ and $h_{n}\left(\varphi\left(a_{n}\right)\right) \rightarrow \infty$ as $n \rightarrow \infty$, we get from (4.8) that $\lim _{\sup _{n \rightarrow \infty}}\left\|g_{n}\right\|_{\mathrm{BMOA}} \leq$ $2 M$. Since $\log \left(2 /\left(1-\left|\varphi\left(a_{n}\right)\right|^{2}\right)\right)=g_{n}\left(\varphi\left(a_{n}\right)\right)$, we may apply the triangle inequality (see (3.16)), Lemma 4.2 and (3.5), to get that

$$
\begin{aligned}
\beta\left(\psi, \varphi, a_{n}\right)= & \left\|\left(\psi \circ \sigma_{a_{n}}-\psi\left(a_{n}\right)\right) g_{n}\left(\varphi\left(a_{n}\right)\right)\right\|_{H^{2}} \\
\leq & \left\|\left(\psi \circ \sigma_{a_{n}}-\psi\left(a_{n}\right)\right) \cdot\left(g_{n} \circ \varphi \circ \sigma_{a_{n}}-g_{n}\left(\varphi\left(a_{n}\right)\right)\right)\right\|_{H^{2}} \\
& +\left\|\left(\psi \circ \sigma_{a_{n}}\right) \cdot g_{n} \circ \varphi \circ \sigma_{a_{n}}-\psi\left(a_{n}\right) g_{n}\left(\varphi\left(a_{n}\right)\right)\right\|_{H^{2}} \\
& +\left\|\psi\left(a_{n}\right)\left(g_{n} \circ \varphi \circ \sigma_{a_{n}}-g_{n}\left(\varphi\left(a_{n}\right)\right)\right)\right\|_{H^{2}} \\
\leq & \left\|g_{n}\right\|_{\mathrm{BMOA}}\left(c\|\psi\|_{*}\left\|\psi \circ \sigma_{a_{n}}-\psi\left(a_{n}\right)\right\|_{H^{2}}\right)^{1 / 2} \\
& +\left\|W_{\psi, \varphi} g_{n}\right\|_{*}+C \alpha\left(\psi, \varphi, a_{n}\right)\left\|g_{n}\right\|_{*} .
\end{aligned}
$$

From (4.6) and (4.1) we get that $\left\|\psi \circ \sigma_{a_{n}}-\psi\left(a_{n}\right)\right\|_{H^{2}} \rightarrow 0$ and $\alpha\left(\psi, \varphi, a_{n}\right) \rightarrow 0$ as $n \rightarrow \infty$. Moreover, since $g_{n} \rightarrow 0$ uniformly on compact subsets of $\mathbb{D}$, we have that $\lim _{n \rightarrow \infty}\left\|W_{\psi, \varphi} g_{n}\right\|_{\text {BMOA }}=0$. Hence $\lim _{n \rightarrow \infty} \beta\left(\psi, \varphi, a_{n}\right)=0$, which contradicts (4.7), so that (4.2) is necessary for the compactness of $W_{\psi, \varphi}$ on BMOA.

Suppose finally that (4.3) does not hold. Then there exist $R \in(0,1)$, $\delta>0, a_{n} \in \mathbb{D}$ and $t_{n} \in(0,1)$, for $n \in \mathbb{N}$, such that $\left|\varphi\left(a_{n}\right)\right| \leq R, t_{n} \rightarrow 1$ as $n \rightarrow \infty$, and

$$
\int_{E\left(\varphi, a_{n}, t_{n}\right)}\left|\left(\psi \circ \sigma_{a_{n}}\right)(\zeta)\right|^{2} d m(\zeta) \geq \delta>0
$$


for all $n \in \mathbb{N}$. By passing to a subsequence if necessary, we may assume that $t_{n}^{n} \rightarrow 1$ as $n \rightarrow \infty$. Let $f_{n}(z)=\sigma_{\varphi\left(a_{n}\right)}(z)^{n}$ for $z \in \mathbb{D}$. Then $\left\|f_{n}\right\|_{\mathrm{BMOA}} \leq$ $3\left\|f_{n}\right\|_{\infty} \leq 3$ and

$$
\begin{aligned}
\left\|W_{\psi, \varphi} f_{n}\right\|_{\mathrm{BMOA}}^{2} & \geq\left\|\left(\psi \circ \sigma_{a_{n}}\right) \cdot f_{n} \circ \varphi \circ \sigma_{a_{n}}\right\|_{H^{2}}^{2} \\
& =\int_{\mathbb{T}}\left|\left(\psi \circ \sigma_{a_{n}}\right)(\zeta)\right|^{2}\left|\left(\sigma_{\varphi(a)} \circ \varphi \circ \sigma_{a}\right)(\zeta)^{n}\right|^{2} d m(\zeta) \\
& \geq \int_{E\left(\varphi, a_{n}, t_{n}\right)}\left|\left(\psi \circ \sigma_{a_{n}}\right)(\zeta)\right|^{2} t_{n}^{2 n} d m(\zeta) \geq t_{n}^{2 n} \delta,
\end{aligned}
$$

for all $n \in \mathbb{N}$. Since $\left|\varphi\left(a_{n}\right)\right| \leq R<1$, the sequence $\left(f_{n}\right)$ converges to 0 uniformly on compact subsets of $\mathbb{D}$ as $n \rightarrow \infty$. Hence $\left\|W_{\psi, \varphi} f_{n}\right\|_{\mathrm{BMOA}} \rightarrow 0$ as $n \rightarrow \infty$. This contradicts (4.9), so that (4.3) is necessary for the compactness of $W_{\psi, \varphi}$ on BMOA. This completes the proof of Step 1.

Step 2. If (4.1), (4.2) and (4.3) hold, then $W_{\psi, \varphi}$ is compact on BMOA.

Proof. Let $\left(f_{n}\right) \subset B_{\mathrm{BMOA}}$ be a sequence of functions such that $f_{n} \rightarrow 0$ uniformly on compact subsets of $\mathbb{D}$. Since $\left(f_{n}\right)$ is a normal family, it suffices to show that $\left\|W_{\psi, \varphi} f_{n}\right\|_{\mathrm{BMOA}} \rightarrow 0$ as $n \rightarrow \infty$ (see e.g. the argument in the proof of [CoM, Proposition 3.11]). For this aim, let $0<\varepsilon \leq 1$. Then, by (4.1) and (4.2), there exists $r \in(0,1)$ such that

$$
\sup _{|\varphi(a)|>r} \max \left\{\alpha(\psi, \varphi, a)+\beta(\psi, \varphi, a),\left(\log \frac{2}{1-|\varphi(a)|^{2}}\right)^{-1 / 2}\right\}<\varepsilon,
$$

and by (4.3), there exists $t \in\left[\frac{1}{2}, 1\right)$ such that

$$
\sup _{|\varphi(a)| \leq r} \int_{E(\varphi, a, t)}\left|\left(\psi \circ \sigma_{a}\right)(\zeta)\right|^{2} d m(\zeta)<\varepsilon^{4} .
$$

Moreover, there is $n_{0} \in \mathbb{N}$ such that

$$
\sup _{w \in Q}\left|f_{n}(w)\right|<\varepsilon \leq 1
$$

for all $n \geq n_{0}$, where $Q:=\overline{r \mathbb{D}} \cup\left\{\sigma_{b}(z) \in \mathbb{D}: b \in \overline{r \mathbb{D}}, z \in \overline{t \mathbb{D}}\right\} \cup\{\varphi(0)\}$ is a compact subset of $\mathbb{D}$.

For any $n \geq n_{0}$, we have that

$$
\begin{aligned}
\left\|W_{\psi, \varphi} f_{n}\right\|_{\mathrm{BMOA}} \leq & \left|\left(W_{\psi, \varphi} f_{n}\right)(0)\right| \\
& +\sup _{|\varphi(a)|>r}\left\|\left(W_{\psi, \varphi} f_{n}\right) \circ \sigma_{a}-\left(W_{\psi, \varphi} f_{n}\right)(a)\right\|_{H^{2}} \\
& +\sup _{|\varphi(a)| \leq r}\left\|\left(W_{\psi, \varphi} f_{n}\right) \circ \sigma_{a}-\left(W_{\psi, \varphi} f_{n}\right)(a)\right\|_{H^{2}} \\
= & : A_{1}+A_{2}+A_{3},
\end{aligned}
$$

where $A_{1}=|\psi(0)| \cdot\left|f_{n}(\varphi(0))\right| \leq \varepsilon\|\psi\|_{\mathrm{BMOA}}$ by (2.2) and (4.12). By the estimate (3.19) (which holds for all functions in $B_{\mathrm{BMOA}}$ ), (3.5) and (2.2), there is a constant $C>0$ such that

$$
\begin{aligned}
A_{2} \leq & \sup _{|\varphi(a)|>r}\left\|\left(\psi \circ \sigma_{a}-\psi(a)\right) \cdot\left(f_{n} \circ \varphi \circ \sigma_{a}-f_{n}(\varphi(a))\right)\right\|_{H^{2}} \\
& +C \sup _{|\varphi(a)|>r}(\alpha(\psi, \varphi, a)+\beta(\psi, \varphi, a)) .
\end{aligned}
$$


Moreover, by Lemma 4.2 we have that

$$
\begin{aligned}
\|\left(\psi \circ \sigma_{a}-\psi(a)\right) & \cdot\left(f_{n} \circ \varphi \circ \sigma_{a}-f_{n}(\varphi(a))\right) \|_{H^{2}} \\
\leq & \left(c\|\psi\|_{*}\left(\log \frac{2}{1-|\varphi(a)|^{2}}\right)^{-1} \beta(\psi, \varphi, a)\right)^{1 / 2} .
\end{aligned}
$$

Since $\sup _{a \in \mathbb{D}} \beta(\psi, \varphi, a)<\infty$ by Theorem 3.1, we get from (4.10) that $A_{2} \leq$ $C_{1} \varepsilon$ for some constant $C_{1}>0$.

We estimate next the term $A_{3}$. Let us abbreviate $F_{n, a}=f_{n} \circ \varphi \circ \sigma_{a}-$ $f_{n}(\varphi(a))$ for $a \in \mathbb{D}$ and $n \geq n_{0}$. Then

$$
\begin{aligned}
A_{3} \leq & \sup _{|\varphi(a)| \leq r}\left\|\left(\psi \circ \sigma_{a}-\psi(a)\right) \cdot f_{n}(\varphi(a))\right\|_{H^{2}} \\
& +\sup _{|\varphi(a)| \leq r}\left\|\left(\psi \circ \sigma_{a}\right) \cdot\left(f_{n} \circ \varphi \circ \sigma_{a}-f_{n}(\varphi(a))\right)\right\|_{H^{2}} \\
\leq & \|\psi\|_{*} \cdot \sup _{|w| \leq r}\left|f_{n}(w)\right|+\left(A_{4}+A_{5}\right)^{1 / 2},
\end{aligned}
$$

where $\sup _{|w| \leq r}\left|f_{n}(w)\right|<\varepsilon$ by (4.12),

$$
A_{4}=\sup _{|\varphi(a)| \leq r} \int_{\mathbb{T} \backslash E(\varphi, a, t)}\left|\left(\psi \circ \sigma_{a}\right)(\zeta) F_{n, a}(\zeta)\right|^{2} d m(\zeta)
$$

and

$$
A_{5}=\sup _{|\varphi(a)| \leq r} \int_{E(\varphi, a, t)}\left|\left(\psi \circ \sigma_{a}\right)(\zeta) F_{n, a}(\zeta)\right|^{2} d m(\zeta) .
$$

In order to estimate $A_{4}$, note that for any $g \in \mathcal{H}(\mathbb{D})$ with $g(0)=0$, it holds that

$$
|g(z)| \leq 2|z| \sup _{|w| \leq t}|g(w)|,
$$

for all $z \in \overline{t \mathbb{D}}$. Indeed, if $g$ is non-zero, then we can define a function $\tilde{g} \in B_{H^{\infty}}$ by setting $\tilde{g}(z)=g(t z) /\left(\sup _{|w| \leq t}|g(w)|\right)$. Since $\tilde{g}(0)=0$, the Schwarz lemma implies for $z \in t \mathbb{D}$ that $|g(\bar{z})|=|\tilde{g}(z / t)|\left(\sup _{|w| \leq t}|g(w)|\right) \leq$ $(|z| / t)\left(\sup _{|w| \leq t}|g(w)|\right)$. This gives (4.15) since $t \geq \frac{1}{2}$ and $g$ is continuous in $\overline{t \bar{D}}$.

Put next $G_{n, a}=f_{n} \circ \sigma_{\varphi(a)}-f_{n}(\varphi(a))$ and $\lambda_{a}=\sigma_{\varphi(a)} \circ \varphi \circ \sigma_{a}$ for $a \in \mathbb{D}$ and $n \geq n_{0}$. Then $G_{n, a}(0)=0$ and $F_{n, a}=G_{n, a} \circ \lambda_{a}$. By applying (4.15) to the functions $G_{n, a}$ we get that

$$
\left|F_{n, a}(\zeta)\right|=\left|G_{n, a}\left(\lambda_{a}(\zeta)\right)\right| \leq 2\left|\lambda_{a}(\zeta)\right| \sup _{|w| \leq t}\left|G_{n, a}(w)\right|,
$$

for all $\zeta \in \mathbb{T}$ such that $\left|\lambda_{a}(\zeta)\right| \leq t$, i.e., for all $\zeta \in \mathbb{T} \backslash E(\varphi, a, t)$. Hence

$$
\begin{aligned}
\int_{\mathbb{T} \backslash E(\varphi, a, t)} \mid & \left|\left(\psi \circ \sigma_{a}\right)(\zeta) F_{n, a}(\zeta)\right|^{2} d m(\zeta) \\
& \leq 4\left(\sup _{|w| \leq t}\left|G_{n, a}(w)\right|\right)^{2} \int_{\mathbb{T}}\left|\left(\psi \circ \sigma_{a}\right)(\zeta) \lambda_{a}(\zeta)\right|^{2} d m(\zeta) .
\end{aligned}
$$

Since

$$
\sup _{|w| \leq t}\left|G_{n, a}(w)\right| \leq \sup _{|w| \leq t}\left|f_{n}\left(\sigma_{\varphi(a)}(w)\right)\right|+\left|f_{n}(\varphi(a))\right| \leq 2 \sup _{w \in Q}\left|f_{n}(w)\right| \leq 2 \varepsilon,
$$


for all $a \in \mathbb{D}$ with $|\varphi(a)| \leq r$ by (4.12), we get that $A_{4} \leq 16 \varepsilon^{2} \sup \left\{\|\left(\psi \circ \sigma_{a}\right)\right.$. $\left.\lambda_{a} \|_{H^{2}}^{2}: a \in \mathbb{D},|\varphi(a)| \leq r\right\}$. Moreover, since

$$
\begin{aligned}
\left\|\left(\psi \circ \sigma_{a}\right) \cdot \lambda_{a}\right\|_{H^{2}} & \leq\left\|\left(\psi \circ \sigma_{a}-\psi(a)\right) \cdot \lambda_{a}\right\|_{H^{2}}+\left\|\psi(a) \cdot \lambda_{a}\right\|_{H^{2}} \\
& \leq\|\psi\|_{*}\left\|\lambda_{a}\right\|_{\infty}+\sup _{a \in \mathbb{D}} \alpha(\psi, \varphi, a)<\infty,
\end{aligned}
$$

we get that $A_{4} \leq C_{2} \varepsilon^{2}$ for some constant $C_{2}>0$.

We finally consider the term $A_{5}$. By the Hölder inequality and (4.11) we have that

$$
\begin{aligned}
A_{5} & \leq \varepsilon^{2} \sup _{|\varphi(a)| \leq r}\left(\int_{E(\varphi, a, t)}\left|\left(\psi \circ \sigma_{a}\right)(\zeta)\right|^{2}\left|F_{n, a}(\zeta)\right|^{4} d m(\zeta)\right)^{1 / 2} \\
& \leq \varepsilon^{2} \sup _{|\varphi(a)| \leq r}\left\|\left(\psi \circ \sigma_{a}\right) \cdot F_{n, a}\right\|_{H^{4}}\left\|F_{n, a}\right\|_{H^{4}} .
\end{aligned}
$$

From (2.1) and (3.3) we get that $\left\|F_{n, a}\right\|_{H^{4}} \leq K_{4}\left\|f_{n} \circ \varphi\right\|_{*} \leq K_{4}$. Moreover, by $(2.1)$ and (4.12) we have that

$$
\begin{aligned}
\sup _{|\varphi(a)| \leq r} & \left\|\left(\psi \circ \sigma_{a}\right) \cdot F_{n, a}\right\|_{H^{4}} \\
\leq & \left.\sup _{|\varphi(a)| \leq r} \|\left(\psi \circ \sigma_{a}\right) \cdot f_{n} \circ \varphi \circ \sigma_{a}-\psi(a) f_{n}(\varphi(a))\right) \|_{H^{4}} \\
& \left.\quad+\sup _{|\varphi(a)| \leq r} \|\left(\psi \circ \sigma_{a}-\psi(a)\right) f_{n}(\varphi(a))\right) \|_{H^{4}} \\
\leq & K_{4}\left\|W_{\psi, \varphi}\right\|+K_{4}\|\psi\|_{*}<\infty .
\end{aligned}
$$

Hence $A_{5} \leq C_{3} \varepsilon^{2}$ for some constant $C_{3}>0$. By combining the above estimates of $A_{4}$ and $A_{5}$ with (4.14) we get that $A_{3} \leq\|\psi\|_{*} \varepsilon+\left(C_{2} \varepsilon^{2}+C_{3} \varepsilon^{2}\right)^{1 / 2}$. Hence, by (4.13), there is a uniform constant $K>0$ such that

$$
\sup _{n \geq n_{0}}\left\|W_{\psi, \varphi} f_{n}\right\|_{\mathrm{BMOA}} \leq A_{1}+A_{2}+A_{3} \leq K \varepsilon
$$

This completes Step 2 and the proof of Theorem 4.1.

It is not difficult to verify that Theorem 4.1 contains as special cases the following characterizations of compactness of multipliers $M_{\psi}$ and composition operators $C_{\varphi}$ on BMOA.

Corollary 4.3. (i) The pointwise multiplier $M_{\psi}$ is compact on BMOA if and only if $\psi(z)=0$ on $\mathbb{D}$.

(ii) The composition operator $C_{\varphi}$ is compact on $\mathrm{BMOA}$ if and only if

$$
\lim _{r \rightarrow 1} \sup _{|\varphi(a)|>r}\left\|\sigma_{\varphi(a)} \circ \varphi \circ \sigma_{a}\right\|_{H^{2}}=0
$$

and, for all $R \in(0,1)$,

$$
\lim _{t \rightarrow 1} \sup _{|\varphi(a)| \leq R} m\left(\left\{\zeta \in \mathbb{T}:\left|\left(\sigma_{\varphi(a)} \circ \varphi \circ \sigma_{a}\right)(\zeta)\right|>t\right\}\right)=0 .
$$

Remark 4.4. Corollary 4.3(ii) is related to the following result due to W. Smith [Sm, Theorem 1.1]. The operator $C_{\varphi}$ is compact on BMOA if and only if

$$
\lim _{r \rightarrow 1} \sup _{|\varphi(a)|>r} \gamma\left(\sigma_{\varphi(a)} \circ \varphi \circ \sigma_{a}\right)=0
$$


where $\gamma$ is defined as in Lemma 3.3, and, for all $R \in(0,1)$,

$$
\lim _{t \rightarrow 1} \sup _{|\varphi(a)| \leq R} m\left(\left\{\zeta \in \mathbb{T}:\left|\left(\varphi \circ \sigma_{a}\right)(\zeta)\right|>t\right\}\right)=0 .
$$

We sketch for completeness a more direct argument which shows that these two characterizations are equivalent. The equivalence of conditions (4.17) and (4.19) can be verified by using the fact that

$$
\frac{1-R}{1+R} \leq \frac{1-\left|\left(\sigma_{\varphi(a)} \circ \varphi \circ \sigma_{a}\right)(\zeta)\right|^{2}}{1-\left|\left(\varphi \circ \sigma_{a}\right)(\zeta)\right|^{2}} \leq \frac{1+R}{1-R},
$$

for all $\zeta \in \mathbb{T}$ and $a \in \mathbb{D}$ such that $\left|\left(\varphi \circ \sigma_{a}\right)(\zeta)\right|<1$ and $|\varphi(a)| \leq R$. These estimates follow easily e.g. from the well-known formula $\left(1-\left|\sigma_{w}(z)\right|^{2}\right) /(1-$ $\left.|z|^{2}\right)=\left|\sigma_{w}^{\prime}(z)\right|$ for $w, z \in \mathbb{D}$ (see $[\mathrm{G}$, p. 3]). The fact that (4.16) implies (4.18) follows from the inequality $\gamma(\lambda) \leq 2\|\lambda\|_{H^{2}}$ which holds for all analytic functions $\lambda: \mathbb{D} \rightarrow \mathbb{D}$ with $\lambda(0)=0$ (see (3.11)). To see that (4.18) implies (4.16) we apply an argument of Smith $[\mathrm{Sm}]$ as follows. Assume that $\gamma(\lambda)<$ $1 / 16$. Then the Littlewood inequality [CoM, p. 33] gives that $N(\lambda, w) \leq$ $\log (1 /|w|)$ if $0<|w|<\gamma(\lambda)^{1 / 4}$, and $N(\lambda, w) \leq \gamma(\lambda)^{1 / 2}$ if $\gamma(\lambda)^{1 / 4} \leq|w|<\frac{1}{2}$, by definition. From these estimates, (3.8) and Lemma 3.3 we get that

$$
\|\lambda\|_{H^{2}}^{2} \leq 4 \int_{0}^{\gamma(\lambda)^{1 / 4}} \log \frac{1}{r} r d r+\frac{2 A\left(\frac{1}{2} \mathbb{D}\right)}{\pi} \gamma(\lambda)^{1 / 2}+\frac{4}{\log 2} \gamma(\lambda),
$$

which yields that $\|\lambda\|_{H^{2}}^{2} \leq C \gamma(\lambda)^{1 / 4}$ for a suitable constant $C>0$.

We leave the remaining details to the interested reader.

\section{Weighted COMPOSITION OPERATORS ON VMOA}

In this section we consider boundedness and compactness of the operators $W_{\psi, \varphi}$ on VMOA, the closed subspace of BMOA consisting of the analytic functions $f \in \mathrm{BMOA}$ such that

$$
\lim _{|a| \rightarrow 1}\left\|f \circ \sigma_{a}-f(a)\right\|_{H^{2}}=0 .
$$

Recall that the space VMOA is in fact the closure of the analytic polynomials in BMOA (see e.g. [Z, §8.4]).

Proposition 5.1. Let $\psi: \mathbb{D} \rightarrow \mathbb{C}$ and $\varphi: \mathbb{D} \rightarrow \mathbb{D}$ be analytic. Then $W_{\psi, \varphi}$ is bounded on VMOA if and only if $W_{\psi, \varphi}$ is bounded on $\mathrm{BMOA}, \psi \in \mathrm{VMOA}$ and

$$
\lim _{|a| \rightarrow 1}|\psi(a)| \cdot\left\|\varphi \circ \sigma_{a}-\varphi(a)\right\|_{H^{2}}=0 .
$$

Proof. Assume first that $W_{\psi, \varphi}$ is bounded on VMOA. Note that for each $a \in \mathbb{D}$ the functions defined in (3.13) and (3.15) belong to VMOA. Thus the argument in the proof Theorem 3.1 shows that

$$
C_{1}\left(\sup _{a \in \mathbb{D}} \alpha(\psi, \varphi, a)+\sup _{a \in \mathbb{D}} \beta(\psi, \varphi, a)\right) \leq\left\|W_{\psi, \varphi}: \mathrm{VMOA} \rightarrow \mathrm{VMOA}\right\|<\infty,
$$


for some constant $C_{1}>0$. Hence $W_{\psi, \varphi}$ is bounded on BMOA. Moreover, we have that $W_{\psi, \varphi}(1)=\psi \in \mathrm{VMOA}$ and $W_{\psi, \varphi}(z)=\psi \varphi \in \mathrm{VMOA}$. Thus

$$
\begin{aligned}
|\psi(a)| \cdot\left\|\varphi \circ \sigma_{a}-\varphi(a)\right\|_{H^{2}} \leq & \left\|\left(\psi \circ \sigma_{a}\right) \cdot \varphi \circ \sigma_{a}-\psi(a) \varphi(a)\right\|_{H^{2}} \\
& +\left\|\left(\psi \circ \sigma_{a}-\psi(a)\right) \cdot \varphi \circ \sigma_{a}\right\|_{H^{2}} \\
\leq & \left\|(\psi \varphi) \circ \sigma_{a}-(\psi \varphi)(a)\right\|_{H^{2}} \\
& +\left\|\psi \circ \sigma_{a}-\psi(a)\right\|_{H^{2}}\|\varphi\|_{H^{\infty}} \rightarrow 0,
\end{aligned}
$$

as $|a| \rightarrow 1$, so that (5.1) holds.

Assume next that $W_{\psi, \varphi}$ is bounded on BMOA, $\psi \in \mathrm{VMOA}$ and (5.1) holds. We will show that $W_{\psi, \varphi}$ is bounded on VMOA. By the closed graph theorem it suffices to show that $W_{\psi, \varphi} f \in$ VMOA where $f \in$ VMOA is arbitrary. Since VMOA is the closure of analytic polynomials in BMOA and $W_{\psi, \varphi}$ is bounded on BMOA, we may assume that $f$ is in fact a polynomial. That is, $f(z)=\sum_{n=0}^{N} c_{n} z^{n}$ for $z \in \mathbb{D}$ for some $N \in \mathbb{N}$ and $c_{0}, \ldots, c_{N} \in \mathbb{C}$. In particular, $M:=\|f\|_{\infty}<\infty$. Let $\varepsilon>0$. By using the assumptions (5.1) and $\psi \in \mathrm{VMOA}$, we may choose a number $r \in(0,1)$ such that $\left\|\psi \circ \sigma_{a}-\psi(a)\right\|_{H^{2}}<$ $\varepsilon / M$ and $|\psi(a)| \cdot\left\|\varphi \circ \sigma_{a}-\varphi(a)\right\|_{H^{2}}<\varepsilon /\left(M N^{2}\right)$ for all $a \in \mathbb{D}$ with $|a| \geq r$. Thus

$$
\begin{aligned}
\left\|\left(W_{\psi, \varphi} f\right) \circ \sigma_{a}-\left(W_{\psi, \varphi} f\right)(a)\right\|_{H^{2}} \leq & \left\|\left(\psi \circ \sigma_{a}-\psi(a)\right) \cdot f \circ \varphi \circ \sigma_{a}\right\|_{H^{2}} \\
& +\left\|\psi(a)\left(f \circ \varphi \circ \sigma_{a}-f(\varphi(a))\right)\right\|_{H^{2}},
\end{aligned}
$$

where $\left\|\left(\psi \circ \sigma_{a}-\psi(a)\right) \cdot f \circ \varphi \circ \sigma_{a}\right\|_{H^{2}} \leq M\left\|\psi \circ \sigma_{a}-\psi(a)\right\|_{H^{2}}<\varepsilon$, for $a \in \mathbb{D}$ with $|a| \geq r$. Since $\left|c_{n}\right|=\left|\int_{0}^{2 \pi} f\left(e^{i \theta}\right) e^{-i n \theta} \frac{d \theta}{2 \pi}\right| \leq M$, we get that

$$
\left\|\psi(a)\left(f \circ \varphi \circ \sigma_{a}-f(\varphi(a))\right)\right\|_{H^{2}} \leq M|\psi(a)| \sum_{n=1}^{N}\left\|\left(\varphi \circ \sigma_{a}\right)^{n}-\varphi(a)^{n}\right\|_{H^{2}} .
$$

From the identity $u^{n}-v^{n}=(u-v) \sum_{k=0}^{n-1} u^{k} v^{n-k-1}$ we obtain the estimate $\left|\left(\varphi \circ \sigma_{a}\right)^{n}(z)-\varphi(a)^{n}\right| \leq n\left|\left(\varphi \circ \sigma_{a}\right)(z)-\varphi(a)\right|$ for $z \in \mathbb{D}$. In particular, we get that

$$
\left\|\psi(a)\left(f \circ \varphi \circ \sigma_{a}-f(\varphi(a))\right)\right\|_{H^{2}} \leq M|\psi(a)| N^{2}\left\|\varphi \circ \sigma_{a}-\varphi(a)\right\|_{H^{2}}<\varepsilon,
$$

for $|a| \geq r$. By combining the above estimates, we get that $W_{\psi, \varphi} f \in$ BMOA and $\left\|\left(W_{\psi, \varphi} f\right) \circ \sigma_{a}-\left(W_{\psi, \varphi} f\right)(a)\right\|_{H^{2}}<2 \varepsilon$ for all $a \in \mathbb{D}$ such that $|a| \geq r$. Consequently $W_{\psi, \varphi} f \in \mathrm{VMOA}$.

It is not difficult to verify that Proposition 5.1 contains as special cases the following known characterizations of boundedness of operators $M_{\psi}$ and $C_{\varphi}$ on VMOA. The case of composition opearators is originally due to Arazy, Fisher and Peetre [AFP, Theorem 12].

Corollary 5.2. (i) The pointwise multiplier $M_{\psi}$ is bounded on VMOA if and only if $\psi \in H^{\infty} \cap$ LMOA.

(ii) The composition operator $C_{\varphi}$ is bounded on VMOA if and only if $\varphi \in \mathrm{VMOA}$.

We consider next compactness of $W_{\psi, \varphi}$ on VMOA. Observe that if $W_{\psi, \varphi}$ is bounded on VMOA, then $W_{\psi, \varphi}$ is compact on VMOA if and only if $W_{\psi, \varphi}$ is compact on BMOA. This results from the facts that $\mathrm{VMOA}^{* *}=\mathrm{BMOA}$ (see e.g. [Z, §8]) and $W_{\psi, \varphi}$ on BMOA is the second adjoint of $W_{\psi, \varphi}$ on VMOA 
(here the argument in [CM, p. 939] for composition operators can also be applied for $\left.W_{\psi, \varphi}\right)$.

We next study compactness of $W_{\psi, \varphi}$ on VMOA more carefully. Our main goal here is to estimate the essential norm

$$
\left\|W_{\psi, \varphi}\right\|_{e}=\inf _{T \in K(\mathrm{VMOA})}\left\|W_{\psi, \varphi}-T\right\|
$$

of an arbitrary weighted composition operator $W_{\psi, \varphi}:$ VMOA $\rightarrow$ VMOA. Here $K(\mathrm{VMOA})$ is the set of all compact linear operators on VMOA. Note in particular that $W_{\psi, \varphi}$ is compact on VMOA if and only if $\left\|W_{\psi, \varphi}\right\|_{e}=0$.

Estimates and formulas for the essential norm of a (weighted) composition operator are known in various settings, see e.g. [S], [M], [MZ], [C̆CZ] and [ČZ2]. However, it seems that such estimates have not appeared in the literature before for (weighted) composition operators on VMOA (or BMOA). Therefore the following result appears to be new also in the special cases of the operators $M_{\psi}$ and $C_{\varphi}$.

Theorem 5.3. Assume that $W_{\psi, \varphi}$ is bounded on VMOA. Then

$$
\left\|W_{\psi, \varphi}\right\|_{e} \sim \limsup _{|a| \rightarrow 1}(\alpha(\psi, \varphi, a)+\beta(\psi, \varphi, a)) .
$$

In particular, $W_{\psi, \varphi}$ is compact on $\mathrm{VMOA}$ if and only if

$$
\lim _{|a| \rightarrow 1} \alpha(\psi, \varphi, a)=0 \quad \text { and } \quad \lim _{|a| \rightarrow 1} \beta(\psi, \varphi, a)=0 .
$$

We need an auxiliary result before the proof of Theorem 5.3. The proof of the following lemma is based on Carleson measure techniques. Similar techniques were used in [T, Theorem 3.11] for characterizing compactness of composition operators on VMOA.

Lemma 5.4. There is a constant $C>0$ such that

$$
\|f\|_{\mathrm{BMOA}} \leq C\left((1-r)^{-5 / 2} \sup _{|z| \leq r}|f(z)|+\sup _{\{a \in \mathbb{D}:|a| \geq r\}}\left\|f \circ \sigma_{a}-f(a)\right\|_{H^{2}}\right),
$$

for all $r \in\left[\frac{1}{2}, 1\right)$ and $f \in \mathrm{BMOA}$.

Proof. We will use the classical fact that a function $f \in \mathcal{H}(\mathbb{D})$ belongs to BMOA if and only if the measure $\mu_{f}$ on $\mathbb{D}$ given by $d \mu_{f}(z)=\left|f^{\prime}(z)\right|^{2}(1-$ $\left.|z|^{2}\right) d A(z) / \pi$ is a Carleson measure (see e.g. [G, §VI.3], [Z, §8]). In fact, there is a constant $C>0$ such that

$$
C^{-1}\|f\|_{*}^{2} \leq \sup _{h \in(0,1]} \sup _{\theta \in[0,2 \pi)} \frac{\mu_{f}(R(h, \theta))}{h} \leq C\|f\|_{*}^{2},
$$

for all $f \in$ BMOA, where $R(h, \theta)$ is the Carleson box $R(h, \theta)=\left\{s e^{i t} \in\right.$ $\mathbb{D}: 1-h \leq s<1,|\theta-t| \leq h\}$ for $h \in(0,1]$ and $\theta \in[0,2 \pi)$.

We establish next the following slightly stronger version of the upper estimate in (5.2). Given $r \in\left[\frac{1}{2}, 1\right)$, put $h_{r}=2(1-r) \in(0,1]$ and

$$
L_{r}=\sup _{\{a \in \mathbb{D}:|a| \geq r\}}\left\|f \circ \sigma_{a}-f(a)\right\|_{H^{2}}^{2} .
$$


Then

$$
\sup _{h \in\left(0, h_{r}\right]} \sup _{\theta \in[0,2 \pi)} \frac{\mu_{f}(R(h, \theta))}{h} \leq C L_{r}
$$

for all $f \in$ BMOA where $C>0$ is a constant. Indeed, one checks that if $h \in\left(0, h_{r}\right], \theta \in[0,2 \pi)$ and $a=\left(1-\frac{h}{2}\right) e^{i \theta}$, then there is a constant $c>0$ such that $\left|\sigma_{a}^{\prime}(z)\right|=\left(1-|a|^{2}\right)|1-\bar{a} z|^{-2} \geq(c h)^{-1}$, for all $z \in R(h, \theta)$. Since $|a|=1-\frac{h}{2} \geq r$, we get that

$$
\sup _{h \in\left(0, h_{r}\right]} \sup _{\theta \in[0,2 \pi)} \frac{\mu_{f}(R(h, \theta))}{h} \leq c \sup _{\{a \in \mathbb{D}:|a| \geq r\}} \int_{\mathbb{D}}\left|\sigma_{a}^{\prime}(z)\right| d \mu_{f}(z) .
$$

The estimate (5.3) follows now from the calculation $\left(1-|z|^{2}\right)\left|\sigma_{a}^{\prime}(z)\right|=1-$ $\left|\sigma_{a}(z)\right|^{2} \leq-2 \log \left|\sigma_{a}(z)\right|=2 N\left(\sigma_{a}, z\right)$ and (3.8).

For $r=\frac{1}{2}$, the proof of the lemma follows from (5.3) and (5.2). Assume next that $r \in\left(\frac{1}{2}, 1\right)$. Let $h \in\left(h_{r}, 1\right]$ and $\theta \in[0,2 \pi)$ be arbitrary. Then it is easy to verify that there are $\theta_{1}, \ldots \theta_{N} \in[0,2 \pi)$ such that $R(h, \theta) \subset$ $\left(\bigcup_{j=1}^{N} R\left(h_{r}, \theta_{j}\right)\right) \cup\left(1-h_{r}\right) \mathbb{D}$, where $N \leq h / h_{r}+1$. By applying (5.3), we get that

$$
\begin{aligned}
\frac{\mu_{f}(R(h, \theta))}{h} & \leq \sum_{j=1}^{N} \frac{\mu_{f}\left(R\left(h_{r}, \theta_{j}\right)\right)}{h}+\frac{\mu_{f}\left(\left(1-h_{r}\right) \mathbb{D}\right)}{h} \\
& \leq 16 N \frac{h_{r}}{h} L_{r}+\frac{1}{h_{r}} \sup _{z \in\left(1-h_{r}\right) \mathbb{D}}\left|f^{\prime}(z)\right|^{2},
\end{aligned}
$$

where $N h_{r} / h \leq 2$. Since $1-h_{r}=2 r-1<r$, an application of the Cauchy integral formula for the derivative gives that $\left|f^{\prime}(z)\right| \leq(1-r)^{-2} \sup _{|z| \leq r}|f(z)|$ for $z \in\left(1-h_{r}\right) \mathbb{D}$. Hence

$$
\sup _{h \in\left(h_{r}, 1\right]} \sup _{\theta \in[0,2 \pi)} \frac{\mu_{f}(R(h, \theta))}{h} \leq 32 L_{r}+\frac{1}{(1-r)^{5}} \sup _{|z| \leq r}|f(z)|^{2} .
$$

Together with (5.3) and (5.2) this proves the lemma.

We are now ready to prove Theorem 5.3.

Proof of Theorem 5.3. We prove first that

$$
C_{1}\left(\limsup _{|a| \rightarrow 1}(\alpha(\psi, \varphi, a)+\beta(\psi, \varphi, a))\right) \leq\left\|W_{\psi, \varphi}\right\|_{e},
$$

for some constant $C_{1}>0$. For this aim, observe that

$$
\limsup _{|a| \rightarrow 1} \sup _{f \in B_{\mathrm{VMOA}}}\left\|(T f) \circ \sigma_{a}-(T f)(a)\right\|_{H^{2}}=0,
$$

for an arbitrary operator $T \in K(\mathrm{VMOA})$. In the case $T=C_{\varphi}$ the proof of (5.5) is contained in [T, Theorem 3.11]. We verify the general case here for completeness. Indeed, if $T$ is compact then $T\left(B_{\mathrm{VMOA}}\right)$ is a relatively compact subset of VMOA. Let $\varepsilon>0$. Then there exist functions $f_{1}, \ldots, f_{n} \in B_{\mathrm{VMOA}}$ such that for every $f \in B_{\mathrm{VMOA}}$ we have $\left\|T f-T f_{j}\right\|_{\mathrm{BMOA}}<\varepsilon$ for some $1 \leq j \leq n$. Consequently, we get that

$$
\sup _{a \in \mathbb{D}}\left\|(T f) \circ \sigma_{a}-(T f)(a)\right\|_{H^{2}} \leq \sup _{a \in \mathbb{D}}\left\|\left(T f_{j}\right) \circ \sigma_{a}-\left(T f_{j}\right)(a)\right\|_{H^{2}}+\varepsilon,
$$


for some $1 \leq j \leq n$. For each $1 \leq j \leq n$, there is $r_{j} \in(0,1)$ such that $\|\left(T f_{j}\right) \circ$ $\sigma_{a}-\left(T f_{j}\right)(a) \|_{H^{2}}<\varepsilon$ whenever $r_{j} \leq|a|<1$. Setting $r=\max \left\{r_{1}, \ldots, r_{n}\right\}$ gives that $\max _{1 \leq j \leq n}\left\|\left(T f_{j}\right) \circ \sigma_{a}-\left(T f_{j}\right)(a)\right\|_{H^{2}}<\varepsilon$ whenever $r \leq|a|<1$. Combining this estimate with (5.6) gives (5.5).

Let $T \in K(\mathrm{VMOA})$ be arbitrary. Then we get from (5.5) that

$$
\begin{aligned}
\| W_{\psi, \varphi} & -T\left\|\geq \sup _{f \in B_{\mathrm{VMOA}}}\right\| W_{\psi, \varphi} f-T f \|_{*} \\
& \geq \limsup _{|a| \rightarrow 1} \sup _{f \in B_{\mathrm{VMOA}}}\left\|\left(\left(W_{\psi, \varphi}-T\right) f\right) \circ \sigma_{a}-\left(\left(W_{\psi, \varphi}-T\right) f\right)(a)\right\|_{H^{2}} \\
& \geq \limsup _{|a| \rightarrow 1} \sup _{f \in B_{\mathrm{VMOA}}}\left\|\left(W_{\psi, \varphi} f\right) \circ \sigma_{a}-\left(W_{\psi, \varphi} f\right)(a)\right\|_{H^{2}},
\end{aligned}
$$

so that

$$
\limsup _{|a| \rightarrow 1} \sup _{f \in B_{\mathrm{VMOA}}}\left\|\left(W_{\psi, \varphi} f\right) \circ \sigma_{a}-\left(W_{\psi, \varphi} f\right)(a)\right\|_{H^{2}} \leq\left\|W_{\psi, \varphi}\right\|_{e} .
$$

Recall that the functions $f_{a}(z)=\sigma_{\varphi(a)}(z)-\varphi(a)$ and $g_{a}(z)=\log (2 /(1-$ $\overline{\varphi(a)} z)$ ) defined in (3.13) and (3.15) satisfy $\sup _{a \in \mathbb{D}}\left\|f_{a}\right\|_{\mathrm{BMOA}} \leq 1$ and $M:=$ $\sup _{a \in \mathbb{D}}\left\|g_{a}\right\|_{*}<\infty$. Moreover, by (3.14) we get that

$$
\alpha(\psi, \varphi, a) \leq 2\left\|\psi \circ \sigma_{a}-\psi(a)\right\|_{H^{2}}+\left\|\left(W_{\psi, \varphi} f_{a}\right) \circ \sigma_{a}-\left(W_{\psi, \varphi} f_{a}\right)(a)\right\|_{H^{2}},
$$

and by (3.16), Lemma 4.2 and (3.5), we get that

$$
\begin{aligned}
\beta(\psi, \varphi, a) \leq & M\left(c\|\psi\|_{*}\left\|\psi \circ \sigma_{a}-\psi(a)\right\|_{H^{2}}\right)^{1 / 2} \\
& +\left\|\left(W_{\psi, \varphi} g_{a}\right) \circ \sigma_{a}-\left(W_{\psi, \varphi} g_{a}\right)(a)\right\|_{H^{2}}+C M \alpha(\psi, \varphi, a) .
\end{aligned}
$$

Note that for each $a \in \mathbb{D}$ in fact $f_{a}, g_{a} \in \mathrm{VMOA}$. Moreover, since $W_{\psi, \varphi}$ is bounded on VMOA, also $\psi \in \mathrm{VMOA}$. Hence we get from the above estimates and (5.7) that

$$
\limsup _{|a| \rightarrow 1}(\alpha(\psi, \varphi, a)+\beta(\psi, \varphi, a)) \leq(1+M+M C)\left\|W_{\psi, \varphi}\right\|_{e} .
$$

This proves (5.4).

We establish next the upper estimate

$$
\left\|W_{\psi, \varphi}\right\|_{e} \leq C_{2} \limsup _{|a| \rightarrow 1}(\alpha(\psi, \varphi, a)+\beta(\psi, \varphi, a)),
$$

for some constant $C_{2}>0$. To this end, define the linear operators $K_{n}$ on VMOA by $\left(K_{n} f\right)(z)=f\left(r_{n} z\right)$ for $n \in \mathbb{N}$ where $r_{n}=(n-1) / n$. Then $K_{n}$ is the composition operator induced by the mapping $\varphi(z)=r_{n} z$, so that $\left\|K_{n}\right\| \leq 1$ and $K_{n}$ is compact (see (3.3) and [MT, Lemma 5.1]). Hence

$$
\left\|W_{\psi, \varphi}\right\|_{e} \leq\left\|W_{\psi, \varphi}-W_{\psi, \varphi} K_{n}\right\|=\sup _{f \in B_{\mathrm{VMOA}}}\left\|W_{\psi, \varphi}\left(I-K_{n}\right) f\right\|_{\mathrm{BMOA}} .
$$

Fix $r \in\left[\frac{1}{2}, 1\right)$ and put $S_{n}=I-K_{n}$ for $n \in \mathbb{N}$. Then Lemma 5.4 gives that

$$
\begin{aligned}
\left\|W_{\psi, \varphi}\right\|_{e} \leq & C(1-r)^{-5 / 2} \sup _{f \in B_{\mathrm{VMOA}}|z| \leq r} \sup _{\psi}\left|\left(W_{\psi, \varphi} S_{n} f\right)(z)\right| \\
& +C \sup _{f \in B_{\mathrm{VMOA}}} \sup _{|a| \geq r}\left\|\left(W_{\psi, \varphi} S_{n} f\right) \circ \sigma_{a}-\left(W_{\psi, \varphi} S_{n} f\right)(a)\right\|_{H^{2}},
\end{aligned}
$$

for all $n \in \mathbb{N}$. 
Note next that

$$
\sup _{f \in B_{\mathrm{BMOA}}} \sup _{|z| \leq r}\left|\left(W_{\psi, \varphi} S_{n} f\right)(z)\right| \rightarrow 0,
$$

as $n \rightarrow \infty$. Indeed, if $\rho=\sup _{|z| \leq r}|\varphi(z)|$ and $R=(1+\rho) / 2$, then $\rho<R<1$ so that the Cauchy integral formula and (2.2) give that

$$
\begin{aligned}
\left|\psi(z)\left(S_{n} f\right)(\varphi(z))\right| & =|\psi(z)|\left|\frac{1}{2 \pi i} \int_{\{|\zeta|=R\}} f(\zeta)\left(\frac{1}{\zeta-\varphi(z)}-\frac{1}{\zeta-r_{n} \varphi(z)}\right) d \zeta\right| \\
& \leq \frac{4}{\log 2}\left(\log \frac{2}{1-r^{2}}\right) \frac{1-r_{n}}{(1-\rho)^{2}}\|\psi\|_{\mathrm{BMOA}}\|f\|_{H^{1}}
\end{aligned}
$$

for $z \in \overline{r \mathbb{D}}$. Since $\|f\|_{H^{1}} \leq\|f\|_{\text {BMOA }}$, this gives (5.10).

Since $\left\|S_{n}\right\| \leq 2$, we get from (5.9) and (5.10) that

$$
\begin{aligned}
\left\|W_{\psi, \varphi}\right\|_{e} \leq & C(1-r)^{-5 / 2} \limsup _{n \rightarrow \infty} \sup _{f \in B_{\mathrm{VMOA}}} \sup _{|z| \leq r}\left|\left(W_{\psi, \varphi} S_{n} f\right)(z)\right| \\
& +C \sup _{n \in \mathbb{N}} \sup _{f \in B_{\mathrm{VMOA}}} \sup _{|a| \geq r}\left\|\left(W_{\psi, \varphi} S_{n} f\right) \circ \sigma_{a}-\left(W_{\psi, \varphi} S_{n} f\right)(a)\right\|_{H^{2}} \\
\leq & 2 C \sup _{g \in B_{\mathrm{VMOA}}|a| \geq r} \sup _{|a|}\left\|\left(W_{\psi, \varphi} g\right) \circ \sigma_{a}-\left(W_{\psi, \varphi} g\right)(a)\right\|_{H^{2}},
\end{aligned}
$$

where $r \in\left[\frac{1}{2}, 1\right)$ is arbitrary. By letting $r \rightarrow 1$, we get that

$$
\left\|W_{\psi, \varphi}\right\|_{e} \leq 2 C \limsup _{|a| \rightarrow 1} \sup _{g \in B_{\mathrm{VMOA}}}\left\|\left(W_{\psi, \varphi} g\right) \circ \sigma_{a}-\left(W_{\psi, \varphi} g\right)(a)\right\|_{H^{2}} .
$$

By the estimate (3.19) (which holds for all $g \in B_{\mathrm{BMOA}}$ ), (3.5) and (2.2), there is a constant $C^{\prime}>0$ such that

$$
\begin{aligned}
\sup _{g \in B_{\mathrm{VMOA}}} \|\left(W_{\psi, \varphi} g\right) \circ & \sigma_{a}-\left(W_{\psi, \varphi} g\right)(a) \|_{H^{2}} \\
\leq & \sup _{g \in B_{\mathrm{VMOA}}}\left\|\left(\psi \circ \sigma_{a}-\psi(a)\right) \cdot\left(g \circ \varphi \circ \sigma_{a}-g(\varphi(a))\right)\right\|_{H^{2}} \\
& +C^{\prime}(\alpha(\psi, \varphi, a)+\beta(\psi, \varphi, a)),
\end{aligned}
$$

where

$$
\begin{aligned}
& \sup _{g \in B_{\mathrm{VMOA}}} \|\left(\psi \circ \sigma_{a}-\psi(a)\right) \cdot\left(g \circ \varphi \circ \sigma_{a}-g(\varphi(a))\right) \|_{H^{2}} \\
& \leq\left(c\|\psi\|_{*}\left\|\psi \circ \sigma_{a}-\psi(a)\right\|_{H^{2}}\right)^{1 / 2}
\end{aligned}
$$

by Lemma 4.2 . Since $\psi=W_{\psi, \varphi}(1) \in \mathrm{VMOA}$, the above estimates and (5.11) give that

$$
\left\|W_{\psi, \varphi}\right\|_{e} \leq C_{2} \limsup _{|a| \rightarrow 1}(\alpha(\psi, \varphi, a)+\beta(\psi, \varphi, a)),
$$

for a suitable constant $C_{2}>0$. This yields (5.8) and completes the proof of Theorem 5.3.

Corollary 5.5. Assume that $\psi \in H^{\infty} \cap \operatorname{LMOA}$ and $\varphi: \mathbb{D} \rightarrow \mathbb{D}$ belongs to VMOA. Then

$$
\left\|M_{\psi}\right\|_{e} \sim\|\psi\|_{\infty}+\limsup _{|a| \rightarrow 1}\left(\log \frac{2}{1-|a|^{2}}\right)\left\|\psi \circ \sigma_{a}-\psi(a)\right\|_{H^{2}}
$$


and

$$
\left\|C_{\varphi}\right\|_{e} \sim \limsup _{|a| \rightarrow 1}\left\|\sigma_{\varphi(a)} \circ \varphi \circ \sigma_{a}\right\|_{H^{2}} .
$$

Remark 5.6. Corollary 5.5 implies in particular that $C_{\varphi}$ is compact on VMOA if and only if $\varphi \in$ VMOA and

$$
\lim _{|a| \rightarrow 1}\left\|\sigma_{\varphi(a)} \circ \varphi \circ \sigma_{a}\right\|_{H^{2}}=0 .
$$

Note that by (3.5) (for $\psi \equiv 1$ ), the condition (5.12) is equivalent to

$$
\lim _{|a| \rightarrow 1} \sup _{f \in B_{\mathrm{VMOA}}}\left\|f \circ \varphi \circ \sigma_{a}-f(\varphi(a))\right\|_{H^{2}}=0 .
$$

Hence $C_{\varphi}$ is compact on VMOA if and only if (5.13) holds. This result is originally due to Tjani [T, Theorem 3.11] (see also [MT, Theorem 4.2], and [BCM, Theorem 3.5] for a similar result).

The compactness of the composition operator $C_{\varphi}$ on VMOA can be characterized in terms of the Nevanlinna counting function. Indeed, if $C_{\varphi}$ is bounded on VMOA, then the argument in Remark 4.4 implies that (5.12) is equivalent to

$$
\lim _{|a| \rightarrow 1} \sup _{w \in \mathbb{D} \backslash\{0\}}|w|^{2} N\left(\sigma_{\varphi(a)} \circ \varphi \circ \sigma_{a}, w\right)=0 .
$$

Hence $C_{\varphi}$ is compact on VMOA if and only if $\varphi \in \mathrm{VMOA}$ and (5.14) holds.

\section{EXAMPLES}

We consider next some examples of bounded and compact weighted composition operators on BMOA and VMOA. Assume that $\psi: \mathbb{D} \rightarrow \mathbb{C}$ and $\varphi: \mathbb{D} \rightarrow \mathbb{D}$ are analytic. Clearly, if both of the operators $M_{\psi}$ and $C_{\varphi}$ are bounded on BMOA, then $W_{\psi, \varphi}$ is bounded on BMOA. If, in addition, either of $M_{\psi}$ or $C_{\varphi}$ is compact, then $W_{\psi, \varphi}$ is compact.

However, it is easy to find analytic mappings $\varphi$ and $\psi$ such that the corresponding weighted composition operator $W_{\psi, \varphi}$ is bounded, but the operator $M_{\psi}$ is not bounded on BMOA. Moreover, there are mappings $\varphi$ and $\psi$ such that $W_{\psi, \varphi}$ is compact, but the operators $M_{\psi}$ and $C_{\varphi}$ are not compact on BMOA.

Example 6.1. Assume that $\psi \in \mathrm{BMOA}$ and $\|\varphi\|_{\infty}<1$. Then $W_{\psi, \varphi}$ is bounded on BMOA.

Proof. Since $W_{\psi, \varphi} f=\psi \cdot(f \circ \varphi)=M_{f \circ \varphi}(\psi)$ and $\psi \in$ BMOA, it suffices to show, by Corollary 3.4, that $\|f \circ \varphi\|_{\infty}$ and $\|f \circ \varphi\|_{*, \log }$ are bounded uniformly in $f \in B_{\mathrm{BMOA}}$. The fact that $\|f \circ \varphi\|_{\infty} \leq C\|f\|_{\mathrm{BMOA}}$ for some constant $C>0$ follows easily from (2.2), since $\|\varphi\|_{\infty}<1$. Moreover, by applying the Cauchy integral formula for the derivative, one finds a constant $C^{\prime}>0$ such that $\left|(f \circ \varphi)^{\prime}(z)\right| \leq C^{\prime}\|f\|_{\text {BMOA. Hence it follows from (3.8) that }}$

$$
\begin{aligned}
\left\|f \circ \varphi \circ \sigma_{a}-f(\varphi(a))\right\|_{H^{2}} & =\left(2 \int_{\mathbb{D}}\left|(f \circ \varphi)^{\prime}(z)\right|^{2} N\left(\sigma_{a}, z\right) \frac{d A(z)}{\pi}\right)^{1 / 2} \\
& \leq C^{\prime}\|f\|_{\mathrm{BMOA}}\left\|\sigma_{a}-a\right\|_{H^{2}},
\end{aligned}
$$

where $\left\|\sigma_{a}-a\right\|_{H^{2}}=\sqrt{1-|a|^{2}}$, so that $\|f \circ \varphi\|_{*, \log } \leq C^{\prime}\|f\|_{\mathrm{BMOA}}$. 
Example 6.2. Let $\psi(z)=1-z$ and $\varphi(z)=(z+1) / 2$. Then neither $M_{\psi}$ nor $C_{\varphi}$ is compact on BMOA, but $W_{\psi, \varphi}$ is compact on VMOA.

Proof. The operators $M_{\psi}$ and $C_{\varphi}$ are not compact on BMOA since $\psi$ is nonzero and $C_{\varphi}$ is not compact even on $H^{2}$ [BCM, Theorem 4.1]. A calculation shows that $\left(\sigma_{\varphi(a)} \circ \varphi \circ \sigma_{a}\right)(z)=\left(2\left(1-|a|^{2}\right) z\right) /\left(4-|a+1|^{2}+(1-\bar{a})^{2} z\right)$, so that

$$
\left\|\sigma_{\varphi(a)} \circ \varphi \circ \sigma_{a}\right\|_{H^{2}}^{2}=\frac{4\left(1-|a|^{2}\right)^{2}}{\left(4-|1+a|^{2}\right)^{2}-|1-a|^{4}}=\frac{1-|a|^{2}}{2(1-\operatorname{Re} a)},
$$

for all $a \in \mathbb{D}$. Since $|1-a|^{2}=1-2 \operatorname{Re} a+|a|^{2}<2(1-\operatorname{Re} a)$, we get that

$$
\alpha(\psi, \varphi, a) \leq \sqrt{1-|a|^{2}} .
$$

Moreover, since $4\left(1-|\varphi(a)|^{2}\right)=1-|a|^{2}+2 \operatorname{Re}(1-a) \geq 2\left(1-|a|^{2}\right)$ and $\left\|\psi \circ \sigma_{a}-\psi(a)\right\|_{H^{2}}=\left\|\sigma_{a}-a\right\|_{H^{2}}=\sqrt{1-|a|^{2}}$, we have that

$$
\beta(\psi, \varphi, a) \leq\left(\log \frac{4}{1-|a|^{2}}\right) \sqrt{1-|a|^{2}} .
$$

Hence $W_{\psi, \varphi}$ is bounded on BMOA, by Theorem 3.1. Clearly $\psi \in$ VMOA. Moreover, since $\varphi \in \mathrm{VMOA}$, we have that

$$
\lim _{|a| \rightarrow 1}|\psi(a)| \cdot\left\|\varphi \circ \sigma_{a}-\varphi(a)\right\|_{H^{2}}=0,
$$

so that $W_{\psi, \varphi}$ is bounded on VMOA, by Proposition 5.1. Since $\alpha(\psi, \varphi, a) \rightarrow 0$ and $\beta(\psi, \varphi, a) \rightarrow 0$ as $|a| \rightarrow 1$, Theorem 5.3 implies that $W_{\psi, \varphi}$ is compact on VMOA.

\section{Comparison results: BMOA and the Bloch space}

In this final section we consider the relationship of boundedness and compactness of weighted composition operators on BMOA and the Bloch space $\mathcal{B}$. It turns out that the boundedness (respectively compactness) conditions on BMOA and $\mathcal{B}$ are quite similar. This quickly leads to a comparison result between weighted composition operators on these spaces. Recall that a function $f \in \mathcal{H}(\mathbb{D})$ belongs to the Bloch space $\mathcal{B}$, if

$$
\|f\|_{\mathcal{B}}=|f(0)|+\sup _{z \in \mathbb{D}}\left|f^{\prime}(z)\right|\left(1-|z|^{2}\right)<\infty
$$

and that $\mathcal{B}_{0}$ is the closed subspace of $\mathcal{B}$ consisting of $f \in \mathcal{B}$ such that

$$
\lim _{|z| \rightarrow 1}\left|f^{\prime}(z)\right|\left(1-|z|^{2}\right)=0 .
$$

Moreover, we have the continuous inclusions BMOA $\subset \mathcal{B}$ and $\mathrm{VMOA} \subset \mathcal{B}_{0}$. For $w \in \mathbb{D}$ and analytic maps $\psi \in \mathcal{B}$ and $\varphi: \mathbb{D} \rightarrow \mathbb{D}$, consider the quantities

$$
\tilde{\alpha}(\psi, \varphi, w)=|\psi(w)|\left|\varphi^{\prime}(w)\right| \frac{1-|w|^{2}}{1-|\varphi(w)|^{2}}
$$

and

$$
\tilde{\beta}(\psi, \varphi, w)=\left(\log \frac{2}{1-|\varphi(w)|^{2}}\right)\left|\psi^{\prime}(w)\right|\left(1-|w|^{2}\right) .
$$

The following result is due to Ohno and Zhao. 
Theorem 7.1 ([OZ, Theorems 1-4]). (i) The operator $W_{\psi, \varphi}$ is bounded on $\mathcal{B}$ if and only if

$$
\sup _{w \in \mathbb{D}} \tilde{\alpha}(\psi, \varphi, w)<\infty \quad \text { and } \quad \sup _{w \in \mathbb{D}} \tilde{\beta}(\psi, \varphi, w)<\infty .
$$

(ii) $W_{\psi, \varphi}$ is compact on $\mathcal{B}$ if and only if

$$
\lim _{r \rightarrow 1} \sup _{|\varphi(w)| \geq r} \tilde{\alpha}(\psi, \varphi, w)=0 \quad \text { and } \quad \lim _{r \rightarrow 1} \sup _{|\varphi(w)| \geq r} \tilde{\beta}(\psi, \varphi, w)=0 .
$$

(iii) $W_{\psi, \varphi}$ is bounded on $\mathcal{B}_{0}$ if and only if $W_{\psi, \varphi}$ is bounded on $\mathcal{B}, \psi \in \mathcal{B}_{0}$ and

$$
\lim _{|w| \rightarrow 1}|\psi(w)|\left|\varphi^{\prime}(w)\right|\left(1-|w|^{2}\right)=0 .
$$

(iv) $W_{\psi, \varphi}$ is compact on $\mathcal{B}_{0}$ if and only if

$$
\lim _{|w| \rightarrow 1} \tilde{\alpha}(\psi, \varphi, w)=0 \quad \text { and } \quad \lim _{|w| \rightarrow 1} \tilde{\beta}(\psi, \varphi, w)=0 .
$$

If the composition operator $C_{\varphi}$ is compact on BMOA, then by a result of M. Tjani [T, Proposition 3.2], it is compact on $\mathcal{B}$ (however, due to a result of W. Smith, the converse of this result does not hold, see [Sm, p. 2722]). Since VMOA $\subset \mathcal{B}_{0}$, the corresponding result holds also for VMOA and $\mathcal{B}_{0}$ in place of BMOA and $\mathcal{B}$ (see also [BCM, Proposition 6.1]). We give next a simple argument which extends these results for the weighted composition operators $W_{\psi, \varphi}$.

Corollary 7.2. (i) If $W_{\psi, \varphi}$ is bounded on BMOA, then $W_{\psi, \varphi}$ is bounded on $\mathcal{B}$.

(ii) If $W_{\psi, \varphi}$ is compact on $\mathrm{BMOA}$, then $W_{\psi, \varphi}$ is compact on $\mathcal{B}$.

(iii) If $W_{\psi, \varphi}$ is bounded on VMOA, then $W_{\psi, \varphi}$ is bounded on $\mathcal{B}_{0}$.

(iv) If $W_{\psi, \varphi}$ is compact on VMOA, then it is compact on $\mathcal{B}_{0}$.

Proof. The Cauchy integral formula gives that $\left|f^{\prime}(0)\right| \leq\|f\|_{H^{2}}$ for every $f \in$ $H^{2}$. Moreover, $\left|\left(f \circ \sigma_{a}-f(a)\right)^{\prime}(0)\right|=\left|f^{\prime}(a)\right|\left(1-|a|^{2}\right)$ and $\left|\left(\sigma_{\varphi(a)} \circ \varphi \circ \sigma_{a}\right)^{\prime}(0)\right|=$ $\left|\varphi^{\prime}(a)\right|\left(1-|a|^{2}\right) /\left(1-|\varphi(a)|^{2}\right)$ for $a \in \mathbb{D}$. Hence $\tilde{\alpha}(\psi, \varphi, a) \leq \alpha(\psi, \varphi, a)$, $\tilde{\beta}(\psi, \varphi, a) \leq \beta(\psi, \varphi, a)$ and $\left|\psi(a)\left\|\varphi^{\prime}(a)\left|\left(1-|a|^{2}\right) \leq\right| \psi(a) \mid \cdot\right\| \varphi \circ \sigma_{a}-\varphi(a) \|_{H^{2}}\right.$. The proof follows now from Theorems 3.1, 4.1, 5.3 and 7.1, and Proposition 5.1 .

Acknowledgements. I thank my supervisor Hans-Olav Tylli for his valuable suggestions and comments.

\section{REFERENCES}

[AFP] J. Arazy, S.D. Fisher and J. Peetre, Möbius invariant function spaces, J. Reine Angew. Math. 363 (1985), 110-145.

[B] A. Baernstein II, Analytic functions of bounded mean oscillation, in: Aspects of contemporary complex analysis (Proc. NATO Adv. Study Inst., Univ. Durham, Durham, 1979), Academic Press, London, 1980, pp. 3-36.

[BCM] P.S. Bourdon, J.A. Cima and A.L. Matheson, Compact composition operators on BMOA, Trans. Amer. Math. Soc. 351 (1999), 2183-2196.

[CM] J.A. Cima and A.L. Matheson, Weakly compact composition operators on VMO, Rocky Mountain J. Math. 32 (2002), 937-951.

[CH] M.D. Contreras and A.G. Hernández-Díaz, Weighted composition operators on Hardy spaces, J. Math. Anal. Appl. 263 (2001), 224-233. 
[CH2] M.D. Contreras and A.G. Hernández-Díaz, Weighted composition operators between different Hardy spaces, Integral Equations Operator Theory 46 (2003), 165188.

[CoM] C.C. Cowen and B.D. MacCluer, Composition operators on spaces of analytic functions, CRC Press, Boca Raton, 1995.

[ČZ] Z̆. Cučković and R. Zhao, Weighted composition operators on the Bergman space, J. London Math. Soc. 70 (2004), 499-511.

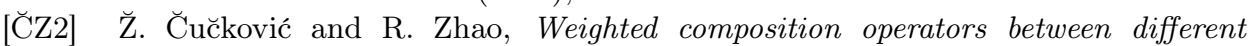
weighted Bergman and Hardy spaces, Illinois J. Math. (to appear).

[FJ] R.J. Fleming and J.E. Jamison, Isometries on Banach spaces: function spaces, Chapman \& Hall/CRC, Boca Raton, 2003.

[G] J.B. Garnett, Bounded analytic functions, Academic Press, New York, 1981.

[MZ] B.D. MacCluer and R. Zhao, Essential norms of weighted composition operators between Bloch-type spaces, Rocky Mountain J. Math. 33 (2003), 1437-1458.

[MT] S. Makhmutov and M. Tjani, Composition operators on some Möbius invariant Banach spaces, Bull. Austral. Math. Soc. 62 (2000), 1-19.

[M] A. Montes-Rodríguez, The essential norm of a composition operator on Bloch spaces, Pacific J. Math. 188 (1999), 339-351.

[OZ] S. Ohno and R. Zhao, Weighted composition operators on the Bloch space, Bull. Austral. Math. Soc. 63 (2001), 177-185.

[OF] J.M. Ortega and J. Fàbrega, Pointwise multipliers and corona type decomposition in BMOA, Ann. Inst. Fourier (Grenoble) 46 (1996), 111-137.

[S] J.H. Shapiro, The essential norm of a composition operator, Ann. of Math. 125 (1987), 375-404.

[S2] J.H. Shapiro, Composition operators and classical function theory, SpringerVerlag, New York, 1993.

[Sm] W. Smith, Compactness of composition operators on BMOA, Proc. Amer. Math. Soc. 127 (1999), 2715-2725.

[St] D.A. Stegenga, Bounded Toeplitz operators on $H^{1}$ and applications of the duality between $\mathrm{H}^{1}$ and the functions of bounded mean oscillation, Amer. J. Math. 98 (1976), 573-589.

[Ste] K. Stephenson, Weak subordination and stable classes of meromorphic functions, Trans. Amer. Math. Soc. 262 (1980), 565-577.

[T] M. Tjani, Compact composition operators on some Möbius invariant Banach spaces, PhD thesis, Michigan State University, 1996.

[WL] M. Wang and P. Liu, Weighted composition operators between Hardy spaces, Math. Appl. (Wuhan) 16 (2003), 130-135.

[Z] K. Zhu, Operator theory in function spaces, Marcel Dekker, New York, 1990.

Department of Mathematics and Statistics, University of Helsinki, P. O. Box 68, Fin-00014 University of Helsinki, Finland

E-mail address: jussi.laitila@helsinki.fi 